\title{
Multidisciplinary Optimization of a Regional Jet Including Advanced Airframe and Engine Technologies
}

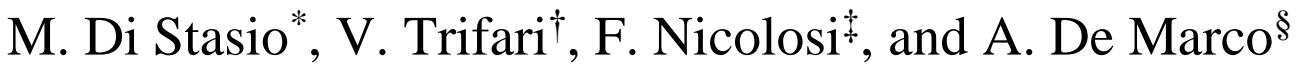 \\ University of Naples Federico II, Naples, 80125, Italy \\ S. Fuhrmann ${ }^{* *}$, and R. Schaber ${ }^{\dagger \dagger}$ \\ MTU Aero Engines AG, Munich, 80995, Germany
}

\begin{abstract}
Aircraft design activities involve several disciplines, whose effects are typically interrelated with each other. The adoption of a multidisciplinary approach is rather fundamental in the design of an aircraft, in order to effectively account all the primary and secondary effects due to changes to one or more design variables. One of the main limitations during the design process of a novel aircraft configuration has always been the lack of communication and exchange of information between aircraft specialists and engine design and performance experts. Even modern software supporting aircraft design activities suffer several limitations in this regard. Moreover, the analysis of innovative aircraft configurations, equipped with the latest airframe technologies, requires the introduction of these technologies and their related multidisciplinary effects in the aircraft design chain. The work performed for the European Clean Sky 2 ADORNO project goes exactly in this direction. An advanced target Y2025+ aircraft model, equipped with advanced under-wing-mounted turbofan engines (designed by MTU Aero Engines AG, topic manager of the project) and innovative airframe technologies, has been designed and analyzed for the second work package of project activities. A UNINA inhouse developed framework for aircraft multidisciplinary analyses and optimizations has been used to carry out all the reported activities. A multi objective optimization, concerning block fuel and direct operating costs, has been carried out including both advanced airframe and engine technologies. The performance in terms of block fuel (thus emissions) of the target aircraft model has been compared with that of a reference Y2014 model, previously designed for the project, to check the achieving of the ambitious objectives of Clean Sky 2 in terms of emissions reductions.
\end{abstract}

\section{Nomenclature}

$\begin{array}{ll}A / C & =\text { aircraft } \\ A C T & =\text { auxiliary central tank } \\ A E A & =\text { all electric architecture } \\ A P I & =\text { application programming interface } \\ B R F & =\text { body reference frame } \\ C F D & =\text { computational fluid dynamics }\end{array}$

*PhD Student, Dept. of Industrial Engineering, mario.distasio@unina.it.

${ }^{\dagger}$ Post-doctoral researcher, Dept. of Industrial Engineering, vittorio.trifari@ unina.it.

¥ Associate Professor, Dept. of Industrial Engineering, fabrizio.nicolosi@ unina.it, AIAA Member.

$\S$ Assistant Professor, Dept. of Industrial Engineering, agostino.demarco@ unina.it.

*** Project Engineer Advanced Programs, Project Engineering, sirka.fuhrmann@mtu.de.

$\dagger$ Consultant Predesign Methodology, Engineering Advanced Programs, reinhold.schaber@mtu.de. 


$\begin{array}{ll}C F R P & =\text { carbon fiber reinforced plastic } \\ D O C & =\text { direct operating costs } \\ D O E & =\text { design of experiments } \\ E C S & =\text { environment control system } \\ E I S & =\text { entry into service } \\ E P N L & =\text { effective perceived noise level } \\ F C S & =\text { flight control system } \\ G A & =\text { genetic algorithms } \\ G T F & =\text { geared turbofan } \\ H L F C & =\text { hybrid laminar flow control } \\ I P S & =\text { ice protection system } \\ I S A & =\text { international standard atmosphere } \\ I T & =\text { information technology } \\ L F L & =\text { landing field length } \\ M D A O & =\text { multidisciplinary design, analysis, and optimization } \\ M E A & =\text { more electric architecture } \\ O B S & =\text { on-board systems } \\ O D E & =\text { ordinary differential equations } \\ O E I & =\text { one engine inoperative } \\ P S O & =\text { particle swarm optimization } \\ R M & =\text { rear-fuselage-mounted engines } \\ S F C & =\text { specific fuel consumption } \\ S S M & =\text { static stability margin } \\ T L A R & =\text { top-level aircraft requirements } \\ T O F L & =\text { take-off field length } \\ T R L & =\text { technology readiness level } \\ U I & =\text { user interface } \\ U M & =\text { under-wing-mounted engines } \\ V_{2} & =\text { minimum take-off safety speed } \\ V C & =\text { variable camber } \\ & \end{array}$

\section{Introduction}

The interaction between aircraft designers and engine manufacturers is a fundamental aspect of the preliminary design process of a new aircraft. A typical issue, which has usually prevented aircraft designers from implementing efficient design loops both in terms of time and costs, is related to the lack of integration between engine and aircraft models inside an iterative aircraft design workflow.

In general, aircraft designers have a comprehensive overview of all aircraft characteristics, as well as their effects on overall aircraft performance. This, together with the development of ever more advanced calculation tools, has allowed to create efficient multidisciplinary analysis and optimization workflows and frameworks to face modern preliminary design activities. Some remarkable examples can be found in Ref. [1-8]. In addition, a general overview of the methodologies typically used for aircraft multi-disciplinary optimizations workflows and their implementations can be found in the work by Martins [9], which introduces a comprehensive description of possible architectures including both monolithic and distributed frameworks.

However, detailed and reliable engine information are usually difficult to retrieve, forcing the designers to make assumptions concerning the engine model or to interact with an engine manufacturer, the latter representing a typical research project scenario in which the iterative exchange of information may lead to a very long preliminary design process. To make this interaction more efficient both in terms of time and costs is the key enabler to deliver innovative and constantly evolving aircraft products.

That is the research context of the ADORNO (Aircraft Design and nOise Rating for regional aircraft) project which complies with the European Union topic JTI-CS2-CfP07-ENG-02-07 (Aircraft design and noise assessment for a regional application) as part of the Clean Sky 2 (CS2) program for Horizon 2020. The project topic leader is the German engine manufacturer MTU Aero Engines, while the University of Naples Federico II (UNINA) is the project coordinator. One of the main goals of the ADORNO project is to allow fast engine optimizations by means of aircraft

\# https://cordis.europa.eu/project/id/821043 
trade factors generated using a multidisciplinary analysis approach. The main technical objective of the project is to provide aircraft requirements (e.g., thrusts, offtakes, etc.) as well as trade factors for specific fuel consumption, engine drag and engine weight on fuel burn for both year 2014 reference aircraft models and CS2 target aircraft models with entry into service expected for 2030 or later.

The reference aircraft platform considered in the framework of the ADORNO project is a regional turbofan similar in terms of top-level aircraft requirements (TLAR) to the Airbus A220-300. The assumed set of requirements has been summarized in Table 1. This set has driven the design of two year 2014 reference A/C models, one with engines mounted under the wing (UM configuration), and one with engines mounted on the rear part of the fuselage [10] (RM configuration).

For both A/C configurations, a multidisciplinary design approach was adopted, including all the disciplines involved in typical analysis process of a novel aircraft configuration. Starting from baseline platforms, statistically generated from the set of TLAR, two aircraft populations, one per aircraft type, were defined by changing some of the most relevant geometrical design parameters such as the wing area, the wing aspect ratio, the wing position, and wing leading edge sweep angle. Then, a multi-disciplinary analysis workflow was set up to collect results in terms of performance and environmental noise. At the end of this procedure, a multi-objective optimization was performed searching for the best $\mathrm{A} / \mathrm{C}$ configurations in terms of mission block fuel and environmental noise. The outcome of this activity were two reference A/C models (Y2014 UM and RM), to be used as the baseline models for comparison in terms of pollutant emissions and environmental noise with respect to the target Y2025+ A/C models, to be designed and analyzed later during the project. The targets in terms of emissions and noise are those set by Clean Sky 2 [11]:

- -20 to $-30 \% \mathrm{CO}_{2}$ emissions ${ }^{\S \S}$,

- -20 to $-30 \% \mathrm{NO}_{\mathrm{x}}$ emissions ${ }^{\S \S}$,

- -20 to $-30 \%$ environmental noise ${ }^{\S}$.

The tool adopted to carry out all the multi-disciplinary analysis and optimization workflows in the framework of the ADORNO project is JPAD [12-17] (Java API for Aircraft Designers), a software for preliminary aircraft design that has been developed at the University of Naples Federico II by the DAF (Design of Aircraft and Flight technologies) research group and by SmartUp Engineering ${ }^{* * *}$, an academic spin-off of the University of Naples.

JPAD takes advantage of the experience gained by the research group members during last years of activities, which have included the participation to several European and non-European projects, related to the development of methodologies and tools supporting aircraft design engineers.

This paper aims at providing a comprehensive overview of the design activities related to the design of a Y2025+ aircraft platform with under-wing engine installation in the framework of the second Work Package (WP) of the ADORNO project. In addition to a general introduction to the JPAD software and the multi-disciplinary analysis and optimization workflows set up for this study, a special focus has been dedicated to the assumptions and the methodology applied to include the effects of advanced airframe and engine technologies in the design framework.

The final section of this paper is dedicated to the description of the results coming from design activities of the ADORNO target Y2025+ UM configuration. This section focuses on the optimization of the target UM A/C, reporting results both in terms of pollutant emissions, which are directly linked to the mission block fuel, and costs. Environmental noise has not been considered in the optimization process since the airframe and the advanced technologies included have only a minor or neglectable impact on the aircraft noise at certification points. In addition, the engine has been assumed to be fixed during all the design activities. Nonetheless, an environmental noise assessment will be performed for the project, to check that Clean Sky 2 objectives in terms of noise reduction have been matched.

Table 1 Set of TLAR assumed to carry out ADORNO A/C design-related activities.

\begin{tabular}{l|c|c}
\multicolumn{1}{c|}{ Attribute } & Value & Unit \\
\hline Design passengers & 140 & \\
\hline Design range & 3100 & $\mathrm{nmi}$ \\
\hline Landing field length & 1509 & $\mathrm{~m}$ \\
\hline Take-off field length & 1890 & $\mathrm{~m}$ \\
\hline Cruise Mach number & 0.78 &
\end{tabular}

\footnotetext{
$\S \S$ With respect to the best aircraft in 2014.
}

**** https://www.smartup-engineering.com/ 


\begin{tabular}{l|c|c} 
Cruise altitude & 37000 & $\mathrm{ft}$ \\
\hline Alternate range & 200 & $\mathrm{nmi}$ \\
\hline Alternate Mach number & 0.65 & \\
\hline Alternate altitude & 20000 & $\mathrm{ft}$ \\
\hline Holding duration & 30 & $\mathrm{~min}$ \\
\hline Holding Mach number & 0.55 &
\end{tabular}

\section{JPAD software}

JPAD is an application programming interface (API), able to support civil transport aircraft designers by building multidisciplinary and simulation-based analysis, as well as optimization workflows. JPAD API is a Java software library collecting classes and utilities to be employed when building software systems running multidisciplinary design, analysis, and optimization (MDAO) tasks.

JPAD comes in different interdependent software modules providing modeling, simulation, and analysis features. An introduction to the possibilities provided by JPAD in terms of aircraft geometric modeling has been provided in Ref. [16], focusing on the capability to automatically generate a fully exportable 3D model.

The JPAD ecosystem is made up of two interconnected software. The first one, named JPAD Modeller, is the basic layer of the JPAD software family, embedding all the modeling capabilities offered by the tool. Coming with a dedicated user interface (UI), it allows to generate and manage a parametrically defined aircraft model (Fig. 1). Each main aircraft component (lifting surfaces, fuselage, cabin, engines, movables) can be customized according to a preselected set of input parameters. These sets have been thoroughly examined and defined to allow an adequate level of flexibility while keeping the total number of manageable input variables the lowest possible. Several update strategies have been implemented to ease the work of the designer and to allow for the modification of more than one input variable at a time, according to precise update rules.

In addition to the basic geometry of the airplane, the user can already manage at this level the aerodynamics of lifting surfaces in terms of airfoil characteristics, by selecting airfoils from a dedicated database (including NACA and supercritical airfoils), or by importing external airfoil lift, drag polar, and pitching moment curves. In this case, JPAD can automatically extract the necessary parameters (e.g., maximum lifting coefficient, lift curve slope, etc.) and provide a comparison between the original curves and the rebuilt ones.

Moreover, engines performance can be managed by means of a dedicated interface featuring the visualization and the calibration of some of the most important engine-related features like thrust, fuel consumption and emissions.

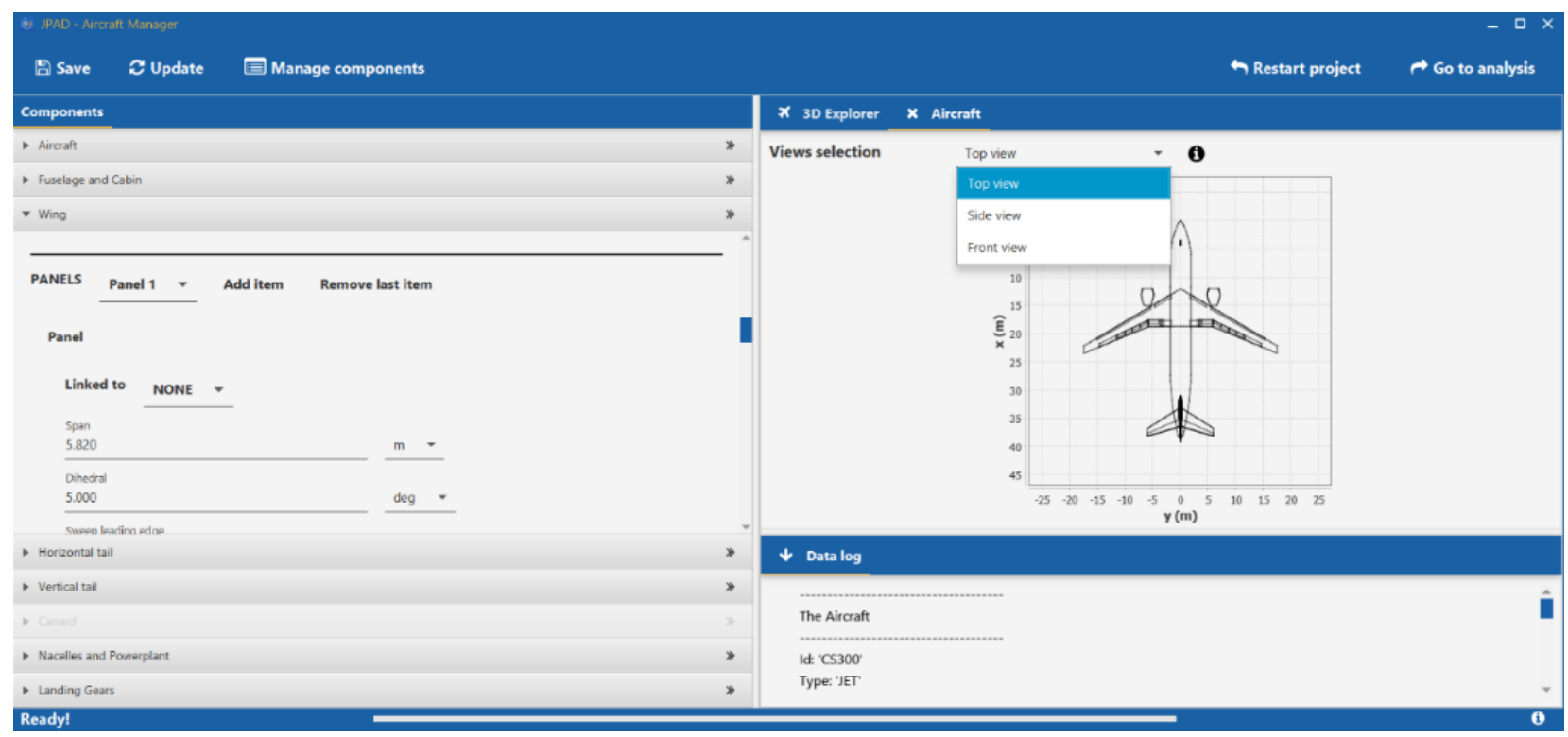

Fig. 1 JPAD Modeller UI, with input tabs reported on the left, and graphical output on the right. 
JPAD MDAO is the second software of the JPAD family, which uses the aircraft model generated by JPAD Modeller to perform multidisciplinary analysis and optimization workflows. Just as JPAD Modeller, JPAD MDAO has been also provided with a dedicated UI, allowing to manage all the input parameters for the analyses, to run them, and to visualize the results.

JPAD MDAO relies on a set of independent calculation modules, which can be easily and conveniently interrelated with each other, to build the desired workflow. The following disciplines have been implemented in the analysis core of JPAD MDAO, and can be included in a preliminary aircraft design workflow:

- Weights - Class-II estimation by means of semiempirical equations available in literature [18-24].

- Balance and Ground Stability - Calculation of the aircraft center of gravity at different weight conditions, the center of gravity of each component, the boarding diagram, aircraft inertia moments and inertia products. A semiempirical approach, based on formulas proposed in Ref. [18] and Ref. [19], has been implemented for the inertias.

- Aerodynamic and Static stability - The aerodynamics module of JPAD allows to calculate the main aerodynamic characteristics concerning lift, drag and moment coefficients, at different operating conditions (e.g., take-off, climb, cruise, and landing) both for the complete aircraft and for each airframe component (wing, tails, fuselage, nacelles). Furthermore, the stability module provides useful data regarding longitudinal and lateral-directional stability. Each analysis is carried out using one (or a mix) of the following approaches: low-fidelity (semiempirical equations from literature), medium-fidelity (vortex lattice method for lifting surface loading, corrected for non-linear effects), high-fidelity (in-house developed surrogate models based on numerical and experimental investigations [25-28]). As for the medium-fidelity approach, JPAD aerodynamics module implements an improved methodology for lift estimation, which overcomes typical limitations of classical vortex methods: the load distribution, obtained by using the NASA-Blackwell approach [29], is modified by taking into account actual airfoil lift characteristics and extending results up to the stall [30].

- Performance - JPAD implements a fully simulation-based performance module. This includes several sub-modules, each supporting a different simulation phase (e.g., take-off, climb, landing) or a specific performance analysis (e.g., payload-range diagram, V-n diagram). Performance of the engines, along with emissions, are included in the analyses by linking each engine of the aircraft to a specific engine dataset at JPAD Modeller level. These datasets must be adequately formatted and include information on net thrust, specific fuel consumption (SFC), and emission indexes for pollutant species, for different flight conditions (altitude, Mach number, ISA deviation) and engine ratings. An adequate set of ordinary differential equations (ODE) has been implemented to handle take-off and landing phases, as well as the simulation of noise trajectories for certification, according to FAR-36 regulations [16][31].

- Emissions - JPAD emissions module allows to estimate pollutant emissions at mission level. Starting from the output coming from performance analyses (i.e., total fuel consumption for each flight phase), the calculation of the total amount of pollutant emitted is performed by considering the emission indexes provided through the engine performance and emissions decks.

- Environmental Noise - JPAD relies on an external tool for the calculation of environmental aircraft noise. This tool, named ATTILA++ (AircrafT noise prediction IncLuding performAnce), has been fully developed by UNINA noise and vibration research group, and has undergone an extensive rewriting, to be interfaced with MTU IT framework within the context of the ADORNO project [32]. ATTILA++ allows to perform custom noise simulation, as well as to calculate effective perceived noise level (EPNL) values at certification points prescribed by FAR-36 regulations. Several ESDU ${ }^{\dagger \dagger \dagger}$ methodologies have been used and adapted in ATTILA++ to include airframe noise contribution [33], as well as sound propagation effects [34-36], and noise shielding effect [37].

- Costs - JPAD costs module allows to perform a breakdown of direct operating costs (DOC), including the following items: capital costs (depreciation, interest, insurance), crew costs (flight and cabin), fuel cost, charges (landing, navigation, ground handling), and direct maintenance (airframe and engines). The semiempirical approaches defined in Ref. [18] and Ref. [38] have been implemented.

An example of workflow with JPAD, involving calculations for all the implemented modules, has been reported in Fig. 2. Nonetheless, the JPAD MDAO framework has been conceived to allow both a complete analysis loop and standalone analyses by means of single calculation modules.

\footnotetext{
ti Engineering Sciences Data Unit, https://www.esdu.com
} 
In the flowchart depicted in Fig. 2 another important feature of the JPAD analysis module is highlighted. The user, in fact, can choose whether to perform a preliminary iterative loop, before even starting the analyses, to calculate the actual amount of fuel needed for the selected mission. The starting point of this iterative loop is a first estimation of the required fuel. Then, a balance analysis is carried out to determine the center of gravity excursion. For each center of gravity, the module for aerodynamics and stability estimates the trimmed drag polar at the following flight conditions: take-off, climb, cruise, and landing. Finally, the performance module uses these results to carry out a mission profile analysis, providing an updated value of the amount of fuel needed to cover the mission. At this point, an iterative process is performed until convergence on the mission fuel mass has been reached. In addition, within the fuel mass iterative loop, an optional iteration can be activated allowing JPAD MDAO to modify the baseline static thrust of each engine to match one of the following requirements:

I. Constant aircraft thrust-to-weight ratio;

II. Take-off field length (TOFL) equal to or lower than a user-defined target value, along with a cruise Mach number equal to or higher than a user-selected target;

III. Same conditions as at point II, but with the addition of a check on the cruise service ceiling, which should be equal to or higher than a user-defined target value.

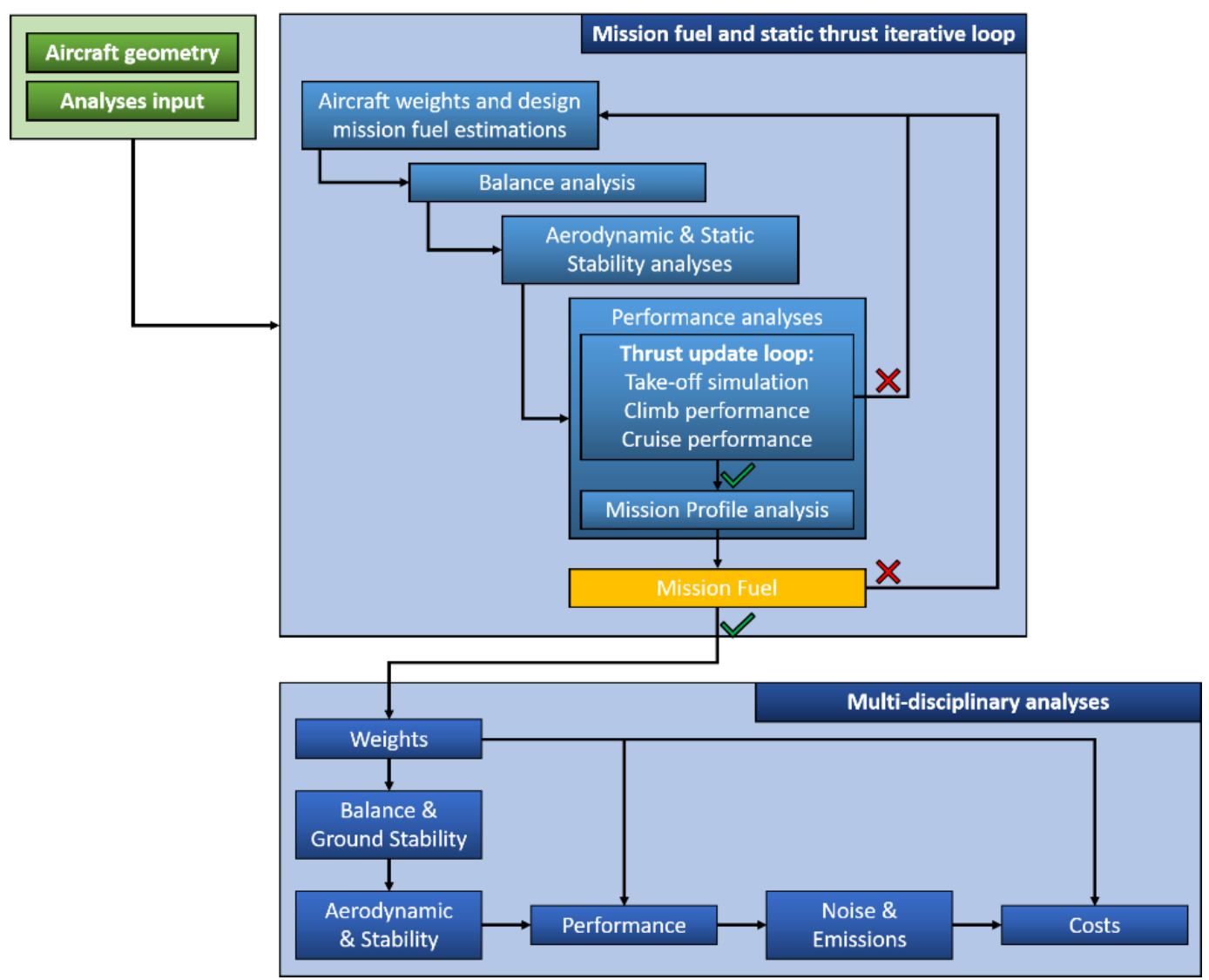

Fig. 2 Complete multidisciplinary analysis workflow implemented by JPAD MDAO.

To effectively support design and optimization tasks, JPAD MDAO includes two additional sub-modules exclusively dedicated to sensitivity analysis and optimization. Those have been widely used for other research activities carried out by the DAF research group as exemplified in Ref. [39].

A typical workflow involving the use of these additional modules has been reported in Fig. 3. Starting from a baseline aircraft (eventually generated with the dedicated modeler) a baseline model for the analyses (i.e., the userdefined set of inputs for the analyses), and a baseline set of operating conditions, the user can define his/her own set of input variables, belonging on principle to any of the abovementioned groups, building a completely custom case study. However, several consistency criteria have been implemented, especially regarding aircraft geometry, to ensure that: 
- There is no simultaneous assignment of eventually conflicting input variables (e.g., there is no possibility to simultaneously assign wing area, wingspan, and wing aspect ratio);

- The obtained A/C models are effectively feasible (e.g., wing and tails connected to the main body, passengers' cabin within fuselage limitations, etc.).

In addition, JPAD $M D A O$ allows the user to choose among several geometrical update strategies, to ease the generation of the aircraft population to be analyzed. These update rules define the way in which aircraft components are automatically updated when modifications are applied to certain aircraft elements. The tool also prevents users from selecting one or more input variables from the set of components subjected to these automatic updates, once that one of these strategies has been effectively selected. For instance, tails geometry can be automatically updated by keeping a constant volumetric ratio (i.e., changes to the geometry of the wing and/or of the fuselage automatically reflect on the horizontal and vertical stabilizers), positions of the engines can be linked to a specific aircraft component (wing or fuselage), main landing gears leg length can be adjusted in automatic to ensure a preselected rotation angle at takeoff, and so on.

The sub-module dedicated to optimizations is based on well-known metaheuristics algorithms, such as genetic algorithms (GA) and particle swarm optimization (PSO) algorithms. The use of metaheuristics algorithms allows to easily manage complex optimization problems with a reduced number of calculations if compared with classical deterministic algorithms (i.e., gradient-based, like Newton-Raphson). In addition, since they do not rely on derivatives but only on objective function values, they are able to easily manage complex and even discontinuous response surfaces. The metaheuristic optimization algorithms implemented by JPAD MDAO come from the free and opensource MOEA Framework ${ }^{+*}$ library.

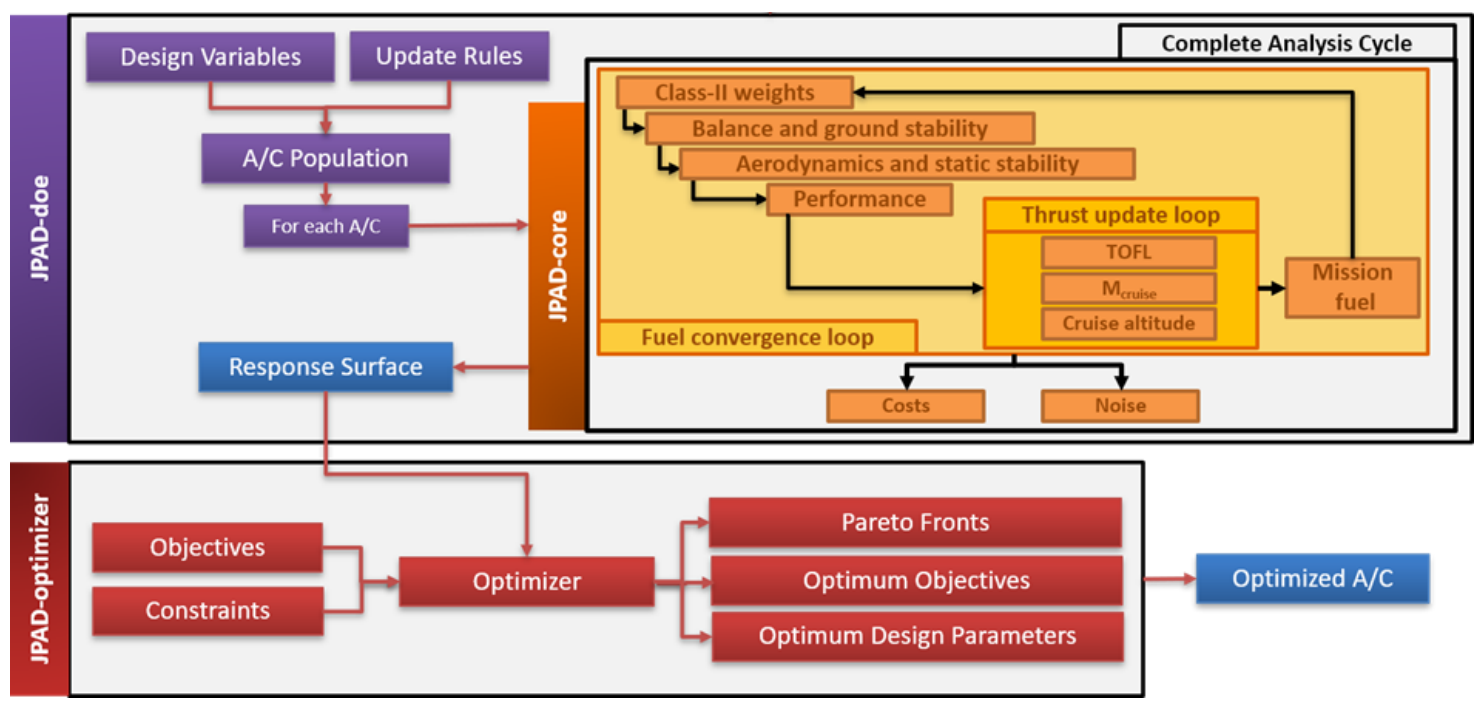

Fig. 3 JPAD MDAO workflow for sensitivity analyses and optimizations.

\section{Airframe technologies selection and characterization}

To match Clean Sky 2 ambitious objectives in terms of emissions and noise reduction, Y2025+ target A/C models should have been designed and analyzed by including the effect of advanced airframe and engine technologies. UNINA has overseen the definition of the set of airframe technologies, whereas MTU has provided a dataset for an advanced geared turbofan (GTF) engine. This section focuses on the description of the set of airframe technologies included in the design framework, and on the assumptions made regarding their impact on the main disciplines involved in the analyses. A separate section has been dedicated to the description of the dataset for the engine.

The first task related to the topic was the selection of plausible technologies, based on expected technology readiness level (TRL) by 2025. This selection was initially carried out starting from the list of airframe technologies to be developed, examined, and demonstrated during the Clean Sky 2 programme and listed in Ref. [11].

Since the Clean Sky 2 development plan is continuously updated, based on the annual results coming from the different work packages, it was hard to produce, just by means of Ref. [11], a list of the most plausible technologies

\#ttp://moeaframework.org/ 
reaching an adequate TRL by 2025, for an expected entry into service (EIS) for the target A/C within the 2030-2035 timeframe. Hence, technologies selection process was also supported by International Air Transport Association ${ }^{\S \S}$ (IATA) aircraft technology roadmap [40-42].

Airframe technologies marked in these documents as evolutionary technologies were selected for the ADORNO advanced $\mathrm{A} / \mathrm{C}$ models. These are technologies that can be fitted to a conventional airplane configuration (i.e., tube and wing) and will be ready to be equipped on the next generation of aircraft with an expected EIS in the next 10-15 years. Thus, the selection has been limited to the following list:

- Hybrid laminar flow control (HLFC), for wing, tails and nacelles.

- Variable camber (VC) on wing, with new high-lift devices.

- Riblets (wing, tails and fuselage).

- More electric on-board systems (OBS) architecture (MEA).

- Advanced materials (advanced alloys and composites).

To implement the set of advanced airframe technologies in the previously described design framework, a strategy based on calibration factors and calibration offsets was developed to include impact on weights, aerodynamics, power off-takes, and costs. For this purpose, a new module was implemented in the JPAD API, dedicated to the definition of the set of calibration factors, along with further user-defined calibrations acting on the ones already implemented in the JPAD code, for each advanced airframe technology.

Dealing with the assumptions on costs, these have been mainly driven by suggestion provided in Ref. [41]. Here, several forecasts in terms of effect on annual operating costs, on-aircraft investment costs, and impact on maintenance are provided for the technologies listed above and many other evolutionary ones. With regards to annual operating costs and on-aircraft investment costs, several tables reported in Ref. [41] provide plausible ranges of variation. These have been used to build and implement a simple but flexible methodology, allowing to perform fast estimations of advanced technologies impact on direct operating costs even at conceptual design phase. Forecasted on-aircraft investment costs reported in Ref. [41] have been assumed as costs to be added to the baseline aircraft price per technology. IATA forecasted variations in terms of annual operating costs, instead, have been retained for a final check, to eventually trim original assumptions. As for maintenance costs, Ref. [41] simply provides an indication in terms of impact by means of six different qualitative indicators: weak detrimental/beneficial effect, mild detrimental/beneficial effect, strong detrimental/beneficial effect. To come up with quantitative assumptions for the maintenance too, the following values have been assumed:

- A weak effect, either beneficial or detrimental, corresponds to a $\pm 1 \%$ variation in maintenance costs with respect to the baseline (i.e., with no advanced technology).

- A mild effect, either beneficial or detrimental, corresponds to a $\pm 10 \%$ variation.

- A strong effect, either beneficial or detrimental, corresponds to a $\pm 25 \%$ variation.

It is important to highlight that assumed values related to the impact on costs have not been used in the following analyses to carry out an accurate estimation of the DOC breakdown for the target A/C models, neither it is one of the main objectives of the project. However, when dealing with advanced technologies, it is quite important to keep an eye on the impact they have on costs, especially when some of the involved technologies might have a strong impact on this discipline, such as HLFC technology. For this reason, in the optimizations and subsequent analyses carried out and described in the following sections, focus has been given both on fuel burn and costs.

With regards to environmental noise, a remarkable impact could not be assumed for any of the advanced airframe technologies involved, at least at certification conditions prescribed by ICAO for the commercial aircraft category (i.e., approach, take-off, sideline).

The following sub-sections provide a summary on the original assumptions made for each technology in terms of impact on weights, aerodynamics, power off-takes and costs, if any. These have been later assumed to equip JPAD with a manager for advanced airframe technologies, which has been subsequently used to eventually trim certain assumptions and to fit input to the analyses to the ADORNO case studies. Detailed information on the actual calibrations for weights, aerodynamics, power off-takes, and costs implemented in JPAD by the advanced airframe technology manager have been also summarized in Tables 2-6. It should be noted that for certain technologies, the JPAD technologies manager provides the possibility to select among three different levels of intensity of a specific effect (namely Low, Medium, and High). Moreover, to furtherly increase flexibility, most of the effects have been paired with an additional calibration attribute, which allows to better trim each single effect. Concerning power off-

$\S \S$ https://www.iata.org/ 
takes and their impact on the mass and on the SFC of the propulsive system, the user has been provided with two possibilities: a) using hard-coded scale factors, based on assumptions from literature, or b) define customized scale factors. The second option has been used to carry out the analyses reported in the following sections, since the MTU dataset for the advanced engine has been provided together with information on dry mass and specific fuel consumption scale factors.

\section{A. Hybrid Laminar Flow Control}

With respect to the assumptions made for impact on aerodynamics, these have been essentially gathered from several reports of actual flight tests, performed both on business and short to medium-haul airliners [43-45]. In particular, Ref. [43] and Ref. [44] provide data on transition delay over wings and tail group thanks to the application of a HLFC system. In general, a transition beyond the $30 \%$ of chord length is predicted.

The effect of this delay has been simulated by means of JPAD aerodynamics module, by manipulating the transition option for the wing airfoils. By setting the transition over the upper and lower surface of the wing beyond the $40 \%$ of chord length, a $40 \%$ reduction in skin friction drag was observed, which was in line with data reported in Ref. [41]. With regards to the impact on the aerodynamics of nacelles, a $35 \%$ reduction in terms of skin friction drag was assumed, considering results reported in Ref. [45].

Hypotheses on the effects on weights have been made starting from data reported in Ref. [46], a study performed by Lockheed to evaluate the application of HLFC technology to a global range military transport aircraft. Here, a breakdown of weight increments had been reported, accounting weight changes for wing, tails, nacelle-engine group, and systems and equipment.

Speaking of power off-takes, indications in terms of power required by the system have been retrieved from Ref. [47], in which a computer performance model for an aircraft similar to the Boeing 757 had been used to study the application of a HLFC system. This study has provided assumptions in terms of power required when applying a HLFC system on different aircraft components, such as tails, wings, and nacelles. To scale these values for different applications, such as the ones involved in ADORNO, they have been divided by the wetted area of their respective aircraft component, in order to come up with scale factors to be used independently from the application.

Finally, dealing with costs, it has been assumed that data reported in Ref. [41] would apply for a full HLFC configuration (i.e., HLFC applied simultaneously on wings, tails, and nacelles). In case of a non-full configuration for HLFC, the following rule has been designed and implemented in the design framework: predicted aircraft price and maintenance increments are divided by the total available wetted area for a HLFC system (wing, tails, and nacelles) and multiplied by the actual wetted area on which HLFC systems are effectively applied.

\section{B. Riblets}

Impact on aerodynamics has been assumed starting from indications provided in Ref. [48-51], which also include results from flight tests and wind tunnel tests. Effects on aircraft weight have been assumed to be negligible, while the same assumptions made for full and non-full HLFC configurations have been applied for aircraft costs.

\section{Variable Camber}

The system concept presented in Ref. [52] (i.e., variable camber with new high-lift devices) has been assumed. With respect to the aerodynamics, assumptions have been made on the impact of this technology starting from the work performed in Ref. [53]. Here, Lyu and Martins have tried to quantify the benefits arising from the adoption of a morphing trailing edge wing for a medium/long range aircraft similar to the Boeing 777-200.

No assumptions have been made in terms of impact on weights and power off-takes. According to Ref. [41], no additional mass should be accounted, especially when dealing with advanced light materials. As for power off-takes, no studies have been retrieved from literature providing information on the required amount of power per flight phase for such a technology.

\section{OBS architecture}

The assumed system architecture for ADORNO target A/C models is the one reported in Ref. [54] as MEA2 (More Electric Aircraft 2), which is characterized as follows:

- A bleed-less configuration: external compressors, powered by the electric system, feed the environmental control system (ECS), while the ice protection system (IPS) is totally electrified.

- The electric system powers the electric pumps of the hydraulic system, which in turn supplies the flight control system (FCS), the actuators for the landing gears, and the wheel brakes. 
Required mechanical power off-takes for such an architecture have been estimated and delivered to MTU for the design of the advanced GTF engine, along with additional required information, about which more details will be provided in the next section.

In addition, two alternatives advanced OBS architectures (the ones indicated in Ref. [54] as MEA1 and AEA) have been also implemented in the JPAD technologies manager module, providing different requirements in terms of power off-takes and bleed air extraction.

For all three configurations, impacts on weights has been assumed starting from information available in Ref. [54]; while no costs assumptions have been included regarding the maintenance contribution. In fact, according to Ref. [41], relevant impact in terms of maintenance costs should not be expected.

\section{E. Advanced materials}

Assumptions on weight reduction linked to the use of carbon fiber reinforced plastic (CFRP) materials for the fuselage and for the wings have been made starting from data reported in Ref. [55] and Ref. [56], respectively. A 25\% weight reduction has been assumed for the fuselage with respect to a conventional full-alloy one, and a $20 \%$ for the wing and for the tail group. A 30\% weight reduction for the landing gears has been assumed starting from data reported in Ref. [57], assuming that advanced Titanium-based alloys are used instead of steel.

Dealing with costs for the use of advanced composites on aircraft main structures, a strategy similar to the one for HLFC and riblets has been implemented, based this time on components weight rather than wetted areas.

Table 2 JPAD advanced airframe technologies manager implemented fudge factors for HLFC.

\begin{tabular}{|c|c|c|c|c|c|c|c|}
\hline HLFC & \multicolumn{7}{|c|}{ Disciplines } \\
\hline & \multicolumn{3}{|c|}{ Aerodynamics } & \multicolumn{4}{|c|}{ Weights } \\
\hline Component & Low & Medium & High & Low & \multicolumn{2}{|c|}{ Medium } & High \\
\hline Wing & $-27 \%$ & $-36 \%$ skin friction drag & $-45 \%$ & - & \multicolumn{2}{|c|}{$+1 \%$} & - \\
\hline Horizontal tail & $-27 \%$ & $-36 \%$ skin friction drag & $-45 \%$ & - & \multicolumn{2}{|c|}{$+2.5 \%$} & - \\
\hline Vertical tail & $-27 \%$ & $-36 \%$ skin friction drag & $-45 \%$ & - & \multicolumn{2}{|c|}{$+2.5 \%$} & - \\
\hline Nacelles & $-35 \%$ & $-35 \%$ skin friction drag & $-35 \%$ & - & \multicolumn{2}{|c|}{$+5.5 \%$} & - \\
\hline Systems & - & - & - & - & \multicolumn{2}{|c|}{$+4 \%$} & - \\
\hline & \multicolumn{7}{|c|}{ Disciplines } \\
\hline & \multicolumn{3}{|c|}{ Power off-takes } & \multicolumn{4}{|c|}{ Costs } \\
\hline Component & Low & Medium & High & & Low & Medium & High \\
\hline Wing & - & $+0.56 \mathrm{~kW} / \mathrm{m}^{2}$ & - & $\mathrm{A} / \mathrm{C}$ price & $+10 \mathrm{M} \$$ & $+50 \mathrm{M} \$$ & $+100 \mathrm{M} \$$ \\
\hline Horizontal tail & - & $+0.44 \mathrm{~kW} / \mathrm{m}^{2}$ & - & Maintenance & $+0 \%$ & $+1 \%$ & $+5 \%$ \\
\hline Vertical tail & - & $+0.77 \mathrm{~kW} / \mathrm{m}^{2}$ & - & & & & \\
\hline Nacelles & - & $+0.76 \mathrm{~kW} / \mathrm{m}^{2}$ & - & & & & \\
\hline Systems & - & - & - & & & & \\
\hline
\end{tabular}

Table 3 JPAD advanced airframe technologies manager implemented fudge factors for Riblets.

\begin{tabular}{|c|c|c|c|c|c|c|c|}
\hline \multirow{3}{*}{$\begin{array}{l}\text { Riblets } \\
\text { Component } \\
\end{array}$} & \multicolumn{7}{|c|}{ Disciplines } \\
\hline & & \multicolumn{3}{|c|}{ Weights } \\
\hline & \multicolumn{3}{|c|}{$\begin{array}{l}\text { Aerodynamics } \\
\text { Medium }\end{array}$} & High & Low & Medium & High \\
\hline Wing & $-5 \%$ & $-7.5 \%$ skin & on drag & $-10 \%$ & - & - & - \\
\hline Horizontal tail & $-5 \%$ & $-7.5 \%$ skin & on drag & $-10 \%$ & - & - & - \\
\hline Vertical tail & $-5 \%$ & $-7.5 \%$ skin & on drag & $-10 \%$ & - & - & - \\
\hline \multirow[t]{3}{*}{ Fuselage } & $-5 \%$ & $-7.5 \%$ skin & on drag & $-10 \%$ & - & - & - \\
\hline & \multicolumn{7}{|c|}{ Disciplines } \\
\hline & \multicolumn{3}{|c|}{ Power off-takes } & \multicolumn{4}{|c|}{ Costs } \\
\hline Component & Low & Medium & High & & Low & Medium & High \\
\hline Wing & - & - & - & $\mathrm{A} / \mathrm{C}$ price & $+1 \mathrm{M} \$$ & $+5 \mathrm{M} \$$ & $+10 \mathrm{M} \$$ \\
\hline Horizontal tail & - & - & - & Maintenance & $+0 \%$ & $+1 \%$ & $+5 \%$ \\
\hline Vertical tail & - & - & - & & & & \\
\hline Fuselage & - & - & - & & & & \\
\hline
\end{tabular}


Table 4 JPAD advanced airframe technologies manager implemented fudge factors for Variable Camber.

\begin{tabular}{|c|c|c|c|c|c|c|c|}
\hline Variable camber & \multicolumn{7}{|c|}{ Disciplines } \\
\hline & \multicolumn{4}{|c|}{ Aerodynamics } & \multicolumn{3}{|c|}{ Weights } \\
\hline Flight condition & Low & \multicolumn{2}{|c|}{ Medium } & High & Low & Medium & High \\
\hline Climb & - & \multicolumn{2}{|c|}{$-4.9 \%$ aerodynamic drag } & - & - & - & - \\
\hline \multirow[t]{3}{*}{ Cruise } & - & \multicolumn{2}{|c|}{$-2.5 \%$ aerodynamic drag } & - & - & - & - \\
\hline & \multicolumn{7}{|c|}{ Disciplines } \\
\hline & \multicolumn{3}{|c|}{ Power off-takes } & \multicolumn{4}{|c|}{ Costs } \\
\hline Flight condition & Low & Medium & High & & Low & Medium & High \\
\hline Climb & - & - & - & $\mathrm{A} / \mathrm{C}$ price & $+1 \mathrm{M} \$$ & $+5 \mathrm{M} \$$ & $+10 \mathrm{M} \$$ \\
\hline Cruise & - & - & - & Maintenance & $+0 \%$ & $+1 \%$ & $+5 \%$ \\
\hline
\end{tabular}

Table 5 JPAD advanced airframe technologies manager implemented fudge factors for OBS architecture.

\begin{tabular}{|c|c|c|c|c|c|c|c|c|}
\hline OBS & & \multicolumn{7}{|c|}{ Disciplines } \\
\hline & & \multicolumn{3}{|c|}{ Aerodynamics } & \multicolumn{4}{|c|}{ Weights } \\
\hline A/C type & & Low & Medium & High & Low & \multicolumn{2}{|c|}{ Medium } & High \\
\hline \multirow{3}{*}{$\begin{array}{l}\text { Reg. jet } \\
90 \text { pax }\end{array}$} & MEA1 & - & - & - & - & \multicolumn{2}{|c|}{$-6.95 \%$ OBS mass } & - \\
\hline & MEA2 & - & - & - & - & \multicolumn{2}{|c|}{$-0.99 \%$ OBS mass } & - \\
\hline & AEA & - & - & - & - & \multicolumn{2}{|c|}{-5.53 OBS mass } & - \\
\hline \multirow{6}{*}{$\begin{array}{c}\text { Med. Haul } \\
160 \text { pax } \\
\end{array}$} & MEA1 & - & - & - & - & \multicolumn{2}{|c|}{-2.51 OBS mass } & - \\
\hline & MEA2 & - & - & - & - & \multicolumn{2}{|c|}{-1.92 OBS mass } & - \\
\hline & AEA & - & - & - & - & \multicolumn{2}{|c|}{-2.95 OBS mass } & - \\
\hline & & & & & & & & \\
\hline & & \multicolumn{7}{|c|}{ Disciplines } \\
\hline & & \multicolumn{3}{|c|}{ Power off-takes } & \multicolumn{4}{|c|}{ Costs } \\
\hline A/C type & & Low & Medium & High & & Low & Medium & High \\
\hline \multirow{3}{*}{$\begin{array}{l}\text { Reg. jet } \\
90 \text { pax }\end{array}$} & MEA1 & - & $-3.80 \%$ & - & $\mathrm{A} / \mathrm{C}$ price & $+100 \mathrm{k} \$$ & $+500 \mathrm{k} \$$ & $+1 \mathrm{M} \$$ \\
\hline & MEA2 & - & $+175.41 \%$ & - & Maintenance & - & - & - \\
\hline & AEA & - & $+171.61 \%$ & - & & & & \\
\hline & & & & & & & & \\
\hline \multirow{3}{*}{$\begin{array}{l}\text { Med. Haul } \\
160 \text { pax }\end{array}$} & MEA1 & - & $-2.27 \%$ & - & & & & \\
\hline & MEA2 & - & $+186.74 \%$ & - & & & & \\
\hline & AEA & - & $+181.65 \%$ & - & & & & \\
\hline
\end{tabular}

Table 6 JPAD advanced airframe technologies manager implemented fudge factors for Materials.

\begin{tabular}{|c|c|c|c|c|c|c|c|}
\hline \multirow{3}{*}{$\begin{array}{l}\text { Advanced materials } \\
\text { CFRP - Components }\end{array}$} & \multicolumn{7}{|c|}{ Disciplines } \\
\hline & \multicolumn{3}{|c|}{ Aerodynamics } & \multicolumn{4}{|c|}{ Weights } \\
\hline & Low & Medium & High & \multicolumn{2}{|l|}{ Low } & Medium & High \\
\hline Wing & - & - & - & \multicolumn{2}{|l|}{-} & $-20 \%$ & - \\
\hline Horizontal tail & - & - & - & \multicolumn{2}{|l|}{-} & $-20 \%$ & - \\
\hline Vertical tail & - & - & - & \multicolumn{2}{|l|}{-} & $-20 \%$ & - \\
\hline Fuselage & - & - & - & \multicolumn{2}{|l|}{-} & $-25 \%$ & - \\
\hline \multicolumn{8}{|l|}{ Ti-based alloys - Components } \\
\hline \multirow[t]{3}{*}{ Landing gears } & - & - & - & \multicolumn{2}{|l|}{-} & $-30 \%$ & - \\
\hline & \multicolumn{7}{|c|}{ Disciplines } \\
\hline & \multicolumn{3}{|c|}{ Power off-takes } & \multicolumn{4}{|c|}{ Costs } \\
\hline CFRP - Components & Low & Medium & High & & Low & Medium & High \\
\hline Wing & - & - & - & $\mathrm{A} / \mathrm{C}$ price & $+1 \mathrm{M} \$$ & $+5 \mathrm{M} \$$ & $+10 \mathrm{M} \$$ \\
\hline Horizontal tail & - & - & - & Maintenance & $+5 \%$ & $+10 \%$ & $+15 \%$ \\
\hline Vertical tail & - & - & - & & & & \\
\hline Fuselage & - & - & - & & & & \\
\hline Ti-based alloys - Components & & & & $\mathrm{A} / \mathrm{C}$ price & $+100 \mathrm{k} \$$ & $+500 \mathrm{k} \$$ & $+1 \mathrm{M} \$$ \\
\hline Landing gears & - & - & - & Maintenance & $+0 \%$ & $+1 \%$ & $+5 \%$ \\
\hline
\end{tabular}




\section{Advanced engine dataset}

MTU oversaw the generation of the dataset (performance and emissions) for an advanced GTF engine to be equipped on ADORNO target A/C models. To enable MTU to perform the design of an advanced turbofan, and to optimize the engine for the selected A/C platform, a trade factor analysis on mission fuel burn has been preliminarily performed by UNINA as initial activity of the fourth Work Package of the ADORNO project.

The trade factor analysis has been carried out on the reference (Y2014) optimized UM A/C, considering the following parameters:

- $\quad$ Single engine dry mass (from the baseline value up to $+300 \mathrm{~kg}$ ),

- Engine SFC (from the baseline value up to $+5 \%$ ).

Each variation for the specified variables has been analyzed individually, to investigate their direct effect. Thus, no cross-correlations have been considered. The design mission fuel of the reference UM A/C has been assumed as the reference value for this study.

For each parameter variation, the aircraft geometry has been updated to ensure that aircraft performance in terms of take-off and landing field lengths, cruise Mach number and time to climb were in line with the baseline model.

The geometrical parameter involved as independent variables in this update process have been the wing area and the wing aspect ratio (if needed). In addition, tails positions and planform parameters have been considered as dependent variables to be modified, to ensure constant volumetric ratios with respect to the reference UM A/C.

The final wing area, for each case, has been selected to match both target take-off and landing field lengths. In case of cruise or climb performance not in line with the target values, or in case of an initial cruise service ceiling not compatible with an operative cruise altitude of $37000 \mathrm{ft}$, the wing aspect ratio has been modified as well, to match those requirements. It is important to remark that, for all the analyses, the overall mission range has been kept constant, and the maximum take-off weight has been adjusted to match the reference design mission.

Fuselage dimensions, as well as landing gears parameters, have been fixed and no modifications have been applied to the position of the wing with respect to the main body. The engine dataset used for the performance analyses has been the same used to carry out the design of the reference $\mathrm{A} / \mathrm{C}$ models.

In addition to the trade factor analysis, definition of the aircraft system architecture (implying power off-takes and customer bleed per flight phase), as well as thrust requirements and maximum dimensions constraints, have been also provided to MTU. This has led to the definition of the final dataset (performance and emissions) for the advanced GTF engine, which has been used to carry out the analyses reported in the following sections.

\section{Airframe technologies fixing and baseline aircraft model definition}

The first step of the design activities needed to define the Y2025+ target UM aircraft for the ADORNO project has been the generation of a baseline parametric model to be used as a reference platform for a full-factorial design of experiments (DOE).

This model would have included both the new engine model from MTU and the possibility to simulate different technology sets. Following the flowchart shown in Fig. 4, the starting point of this activity has been to setup and to trim all calibration factors, made available inside the JPAD technologies manager module, to check if the results for each technology in terms of block fuel reduction and annual operating costs variation were in line with the ranges provided by IATA in Ref. [41]. Thus, assuming an initial set of calibration factors and effect magnitude levels (explained in the previous section), an iterative process has been carried out obtaining the final trimmed set of calibrations reported in Tables 7-11.

It must be noted that effects related to the impact of advanced on-board systems architectures on the cruise SFC have been neglected since those had been already considered in the new engine performance dataset provided by MTU. In addition, having assumed the A220-300 as the reference aircraft model for the design of the Y2014 reference UM aircraft, all advanced materials effects related to lifting surfaces have also been neglected, since the reference aircraft already makes an extensive use of advanced composite materials in the wings and in the empennages. Composite materials are also used for the rear portion of the fuselage of the A220-300, thus, to consider the effect of a full composite fuselage for the ADORNO target aircraft, a calibration factor of 0.6 has been assumed for the fuselage weight calibration of Table 11, thus lowering the hard-coded $25 \%$ weight reduction to $-18.75 \%$. 


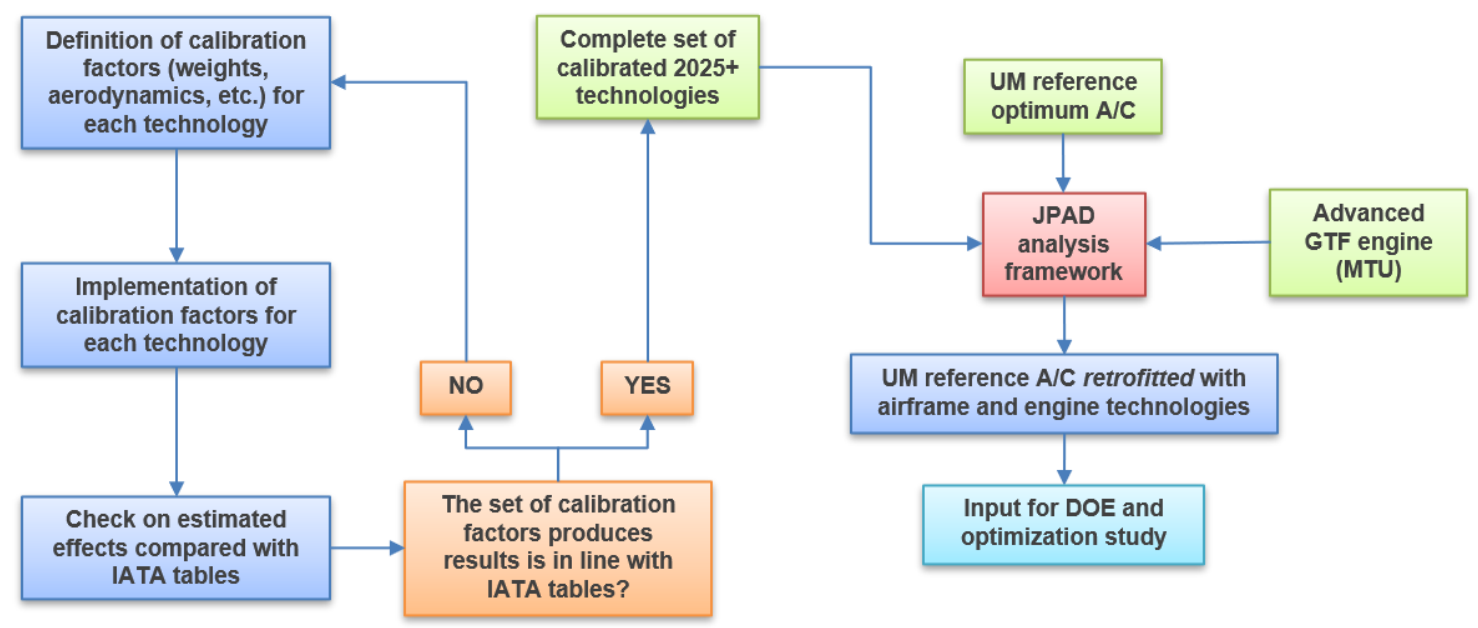

Fig. 4 Flowchart representing the generation process of the baseline aircraft to be used for the MDAO.

Table 7 Final set of calibration factors and effects levels for HLFC. A calibration equal to 1.0 means full estimated effect.

\begin{tabular}{|c|c|c|}
\hline \multicolumn{3}{|c|}{ HLFC Effects } \\
\hline \multirow{3}{*}{ Wing } & Weights & Effect calibration $=1.0$ \\
\hline & Aerodynamics & $\begin{array}{l}\text { Effect level }=\text { HIGH } \\
\text { Effect calibration }=1.2\end{array}$ \\
\hline & Mechanical power off-takes effect on cruise SFC & Effect calibration $=1.0$ \\
\hline \multirow{3}{*}{ Horizontal tail } & Weights & Effect calibration $=1.0$ \\
\hline & Aerodynamics & $\begin{array}{l}\text { Effect level }=\text { HIGH } \\
\text { Effect calibration }=1.1\end{array}$ \\
\hline & Mechanical power off-takes effect on cruise SFC & Effect calibration $=1.0$ \\
\hline \multirow{3}{*}{ Vertical tail } & Weights & Effect calibration $=1.0$ \\
\hline & Aerodynamics & $\begin{array}{l}\text { Effect level = HIGH } \\
\text { Effect calibration }=1.1\end{array}$ \\
\hline & Mechanical power off-takes effect on cruise SFC & Effect calibration $=1.0$ \\
\hline \multirow{3}{*}{ Nacelles and powerplant } & Weights & Effect calibration $=1.0$ \\
\hline & Aerodynamics & Effect calibration $=1.1$ \\
\hline & Mechanical power off-takes effect on cruise SFC & Effect calibration $=1.0$ \\
\hline \multicolumn{2}{|r|}{ Systems weights } & Effect calibration $=1.0$ \\
\hline \multicolumn{2}{|r|}{ Aircraft price } & $\begin{array}{l}\text { Effect level }=\text { LOW } \\
\text { Effect calibration }=1.0\end{array}$ \\
\hline \multicolumn{2}{|r|}{ Maintenance costs } & $\begin{array}{l}\text { Effect level }=\text { HIGH } \\
\text { Effect calibration }=1.0\end{array}$ \\
\hline
\end{tabular}

Table 8 Final set of calibration factors and effects levels for riblets. A calibration equal to 1.0 means full estimated effect.

\begin{tabular}{|c|l|}
\hline \multicolumn{2}{|c|}{ Riblets effects } \\
\hline Wing aerodynamics & $\begin{array}{l}\text { Effect level = MEDIUM } \\
\text { Effect calibration = 1.0 }\end{array}$ \\
\hline \multirow{2}{*}{ Horizontal tail aerodynamics } & $\begin{array}{l}\text { Effect level = MEDIUM } \\
\text { Effect calibration = 1.0 }\end{array}$ \\
\hline \multirow{2}{*}{ Vertical tail aerodynamics } & $\begin{array}{l}\text { Effect level = MEDIUM } \\
\text { Effect calibration = 1.0 }\end{array}$ \\
\hline \multirow{2}{*}{ Fuselage aerodynamics } & $\begin{array}{l}\text { Effect level = MEDIUM } \\
\text { Effect calibration = 1.0 }\end{array}$ \\
\hline Aircraft price & $\begin{array}{l}\text { Effect level = MEDIUM } \\
\text { Effect calibration }=1.0\end{array}$ \\
\hline Maintenance costs & $\begin{array}{l}\text { Effect level = HIGH } \\
\text { Effect calibration }=1.0\end{array}$ \\
\hline
\end{tabular}


Table 9 Final set of calibration factors and effects levels for wing variable camber trailing edge devices. A calibration equal to 1.0 means full estimated effect.

\begin{tabular}{|c|c|}
\hline \multicolumn{2}{|c|}{ Wing variable camber trailing edge devices effects } \\
\hline Aircraft aerodynamics (climb condition) & Effect calibration $=1.0$ \\
\hline Aircraft aerodynamics (cruise condition) & Effect calibration $=1.0$ \\
\hline Aircraft price & $\begin{array}{l}\text { Effect level }=\text { LOW } \\
\text { Effect calibration }=1.0\end{array}$ \\
\hline Maintenance costs & $\begin{array}{l}\text { Effect level }=\text { HIGH } \\
\text { Effect calibration }=0.75\end{array}$ \\
\hline
\end{tabular}

Table 10 Final set of calibration factors and effects levels for advanced on-board systems architecture effects. A calibration equal to 1.0 means full estimated effect.

\begin{tabular}{|c|l|}
\hline \multicolumn{2}{|c|}{ Advanced on-board systems architectures effects } \\
\hline Powerplant weight & Effect calibration $=1.0$ \\
\hline Aircraft price & $\begin{array}{l}\text { Effect level }=\text { LOW } \\
\text { Effect calibration }=1.0\end{array}$ \\
\hline Mechanical power off-takes effect on cruise SFC & Effect calibration $=0.0$ \\
\hline Bleed-less configuration effect on cruise SFC & Effect calibration $=0.0$ \\
\hline
\end{tabular}

Table 11 Final set of calibration factors and effects levels for advanced materials. A calibration equal to 1.0 means full estimated effect.

\begin{tabular}{|c|l|}
\hline \multicolumn{2}{|c|}{ Advanced materials effects } \\
\hline Wing weight & Effect calibration $=0.0$ \\
\hline Horizontal tail weight & Effect calibration $=0.0$ \\
\hline Vertical tail weight & Effect calibration $=0.0$ \\
\hline Fuselage weight & Effect calibration $=0.75$ \\
\hline Landing gears weight & Effect calibration $=1.0$ \\
\hline Aircraft price & $\begin{array}{l}\text { Effect level }=\text { LOW } \\
\text { Effect calibration }=1.0\end{array}$ \\
\hline Maintenance costs & $\begin{array}{l}\text { Effect level }=\text { LOW } \\
\text { Effect calibration }=0.5\end{array}$ \\
\hline
\end{tabular}

With the fully trimmed set of technologies calibration factors and effects levels, the second step in the definition of the Y2025+ UM target aircraft baseline model has been the installation of the new advanced engine.

The new engine provided by MTU is characterized by an increased bypass ratio and overall pressure ratio with respect to the Y2014 reference engine. The larger fan diameter, maximum nacelle diameter and overall length have required to apply some geometrical modifications to the aircraft, enabling for a feasible installation. In fact, these modifications have been applied to comply with all ground stability and ground operation limitations, as well as to provide an adequate amount of ground clearance, a correct landing gears positioning, and the possibility to easily retract the main landing gear inside the wing. A visual comparison between the initial aircraft model and the updated aircraft model has been provided in Fig. 5.

This update process has been mainly driven by the need to not lose the beneficial effect provided by the lower cruise SFC value of the new engine. Thus, several attempts have been made to define the best possible set of modifications reported in Table 12.

Table 12 Geometrical modifications applied to the reference aircraft for the installation of the new engine.

\begin{tabular}{|l|c|c|}
\hline & Previous baseline A/C & Updated baseline A/C \\
\hline Engine vertical offset from the wing attachment & $-1.3 \mathrm{~m}$ & $-1.4 \mathrm{~m}$ \\
\hline Main landing gear leg length & $2.56 \mathrm{~m}$ & $2.85 \mathrm{~m}$ \\
\hline Main landing gear wheel track & $6.73 \mathrm{~m}$ & $6.78 \mathrm{~m}$ \\
\hline
\end{tabular}



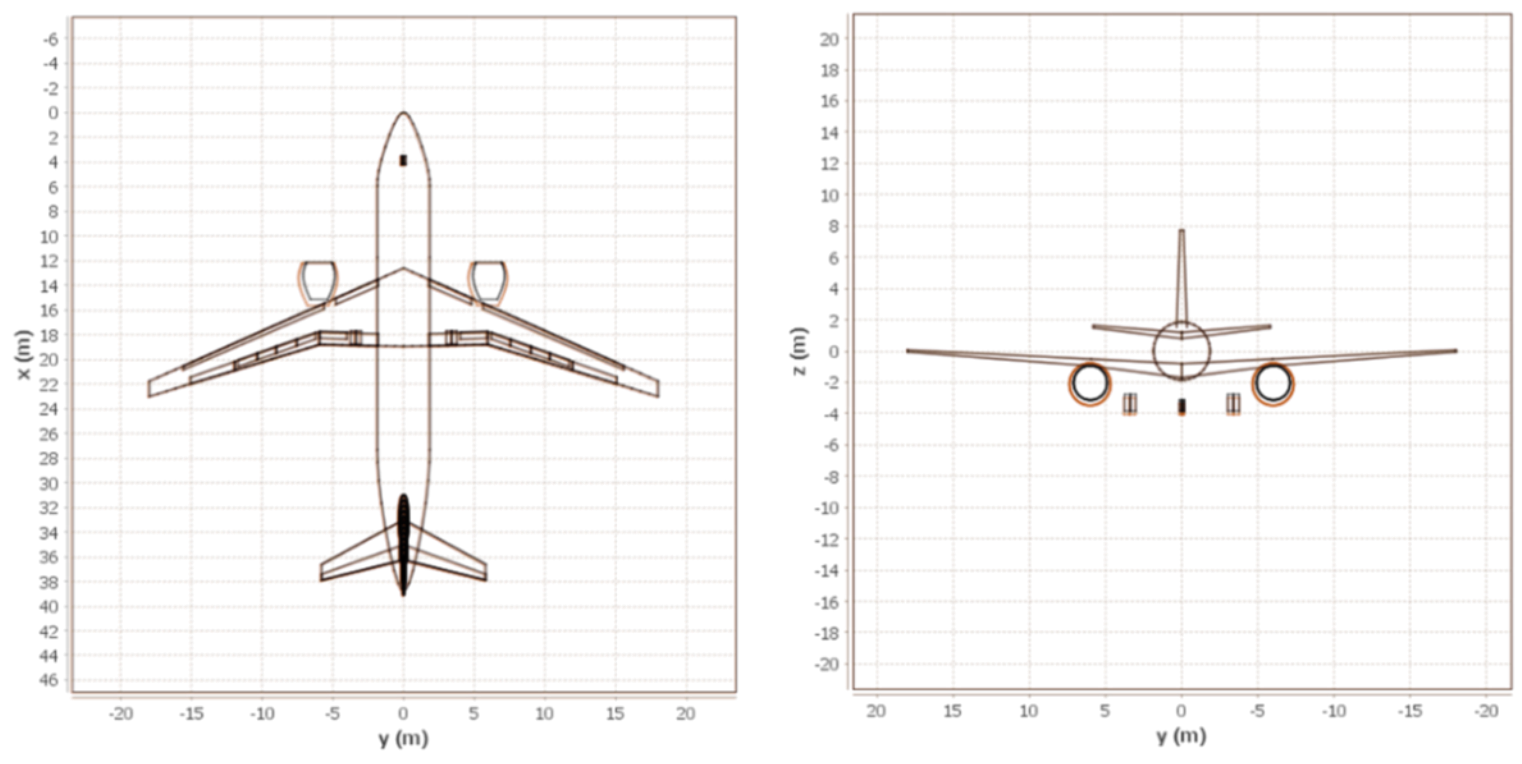

- Reference 2014 ADORNO UM A/C with geometrical modifications

- Reference 2014 ADORNO UM A/C

Fig. 5 JPAD comparison between the initial aircraft and the updated baseline aircraft to install the new engine provided by MTU.

\section{Optimization strategy and results}

Starting from the updated baseline UM aircraft model described in the previous section, a complete MDAO process has been carried out to investigate the effects on mission-related objectives, such as the block fuel and the total DOC, of both the selected set of advanced airframe technologies and some main aircraft geometrical parameters, like the wing area and the wing aspect ratio. All calculations and results reported in this section have been obtained by means of the design framework JPAD described in the previous sections.

The multidisciplinary optimization of the target UM aircraft model has been carried out by following a two-step approach. Firstly, a single-objective optimization on the mission block fuel has been performed, implementing the procedure shown in Fig. 3 for each of the 336 advanced airframe technologies combinations reported in Table 13. Here, the reduced number of combinations concerning the use of advanced composites materials has been already explained in the previous section; while, with regards to the remaining airframe technologies, a preliminary selection, aimed at considering only the most relevant combinations, has been performed to reduce the total number of permutations. This has drastically reduced the overall computational time.

To generate the aircraft population for each of the 336 optimizations, wing planform parameters, represented by the wing area and the wing aspect ratio, have been included in the set of independent design variables. No variations have been applied to the geometry of the fuselage, to be sure to comply with prescribed top-level requirements in terms of accommodation, neither to the relative position of the wing with respect to the fuselage, or the positions of the engines with respect to the wing (i.e., spanwise nondimensional position has been kept constant and equal to the baseline value, as well as the deltas between engine longitudinal and vertical apex and the wing leading edge).

In addition, the same main landing gear leg length of the baseline $\mathrm{A} / \mathrm{C}$ model has been considered for all the performed analyses. It is important to remark that variations just to the wing planform geometry have also implied modifications to other aircraft main and secondary components, according to a pre-selected update strategy. The overall set of modifications to the aircraft geometry can be summarized as follows:

- The wing aspect ratio and wing area have been changed from $-20 \%$ to $+20 \%$ of their baseline values, 11.79 and $109.76 \mathrm{~m}^{2}$ respectively, with 5 equally spaced values. A total of 25 permutations have been considered and for each of them panel chords (inner and outer) and spans have been subsequently updated, so that chords have been multiplied by the ratio between current planform surface and baseline surface, while spans have been updated by keeping the same ratios with respect to the overall span of the baseline configuration. 
- The wing fuel tank dimensions have been updated according to the ratio between the current and the baseline planform areas.

- $\quad$ Planform geometries and positions in the body reference frame (BRF) of the horizontal and vertical tails have been automatically updated to keep the same volumetric ratios and the same aspect ratios of the baseline model.

- Dimensions and positions of the control surfaces and high-lift devices have been automatically updated too, by keeping the same definitions in terms of nondimensional parameters (e.g., inner and outer spanwise positions along lifting surfaces, as well as chordwise position and chord ratios) of the baseline A/C model.

In additions to these geometry updates, it is important to remark that, depending on the implemented set of technologies, the provided baseline engine has been updated in terms of SFC and dry mass, by using scale factors for power off-takes provided by MTU. No thrust update strategies (i.e., the ones reported in the diagram of Fig. 3) have been applied to match specific mission requirements.

For each combination of airframe technologies, a full-factorial design of experiments has been carried out, leading to 336 different response surfaces, with a total of 8400 analyzed aircraft. These have successively fed the JPAD MDAO sub-module dedicated to optimizations to perform 336 different single-objective optimizations on block fuel. In addition to the response surfaces, the sub-module for optimizations has been also provided with a set of constraints, to ensure the feasibility of each of the optimum A/C produced. The set of constraints can be summarized as follows.

- All ground stability and ground operation checks should be successfully passed:

○ Possibility to rotate the aircraft at landing and take-off for a given rotation angle (i.e., the one given by the selected and fixed main landing gear leg length);

- Engines clearance from ground is in line with the minimum limitations from FAR regulations;

○ The bank angle during ground operations is always lower than the maximum allowable turning bank angle;

- The sideways turnover angle is lower than the maximum allowable value of $63^{\circ}$ [58];

- The turning radius is lower than the maximum allowable value prescribed by FAA [59];

○ Landing gears positions complies with limitations estimated, as suggested in Ref. [19].

- The static stability margin (SSM) at the most aft center of gravity position ensures aircraft longitudinal stability (i.e., negative SSM).

- No tail strike during the take-off simulation.

- No tail or nose strike during the landing simulation.

- The minimum take-off safety speed $\left(\mathrm{V}_{2}\right)$ during one engine inoperative (OEI) take-off is at least equal to the aircraft take-off stall speed times 1.13 .

- The cruise altitude (flat cruise) is above $35000 \mathrm{ft}$, with a $2000 \mathrm{ft}$ tolerance with respect to the prescribed TLAR.

- The take-off field length is lower than $1950 \mathrm{~m}$, with a $60 \mathrm{~m}$ tolerance with respect to the value prescribed by the TLAR.

In addition to these constraints, other two have been alternatively switched on:

- The overall wing span is below the current limitation $(36 \mathrm{~m})$ prescribed by ICAO Annex 14 for the C aircraft category.

- The mission fuel mass is lower than the maximum storable in the wing fuel tank.

The last constraint has been slightly softened, when switched off, by assuming to allocate an auxiliary central tank (ACT) in the lower deck, allowing to board an additional fuel mass equal to $2132 \mathrm{~kg}$. The dimensions of this additional tank have been derived from those for the A320 and have been appropriately scaled. With this set of constraints, and by considering all the possible permutations, four different single-objective optimizations on block fuel have been carried per airframe technology set, leading to a total of 1344 optimizations. This first step in the optimization process towards the final UM target aircraft of ADORNO has been summarized in Fig. 6. 
Table 13 Technologies combinations considered in the optimization study.

\begin{tabular}{|c|c|c|c|c|c|}
\hline & \multicolumn{5}{|c|}{ TECHNOLOGIES } \\
\hline & HLFC & Riblets & $\begin{array}{l}\text { Variable } \\
\text { Camber }\end{array}$ & $\begin{array}{c}\text { OBS } \\
\text { Architecture }\end{array}$ & $\begin{array}{l}\text { Advanced } \\
\text { Materials }\end{array}$ \\
\hline \multirow{7}{*}{ 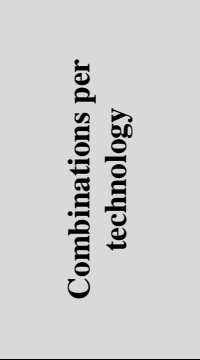 } & None & None & None & MEA2 & None \\
\hline & Wing & Wing & Wing & & Fuselage \\
\hline & Tails & Tails & & & Landing gears \\
\hline & Wing+Tails & Fuselage & & & LG+Fuselage \\
\hline & Tails+Nacelles & Wing+Tails & & & \\
\hline & Wing+Tails+Nacelles & Tails+Fuselage & & & \\
\hline & & Wing+Tails+Fuselage & & & \\
\hline $\begin{array}{l}336 \text { total } \\
\text { combinations }\end{array}$ & 6 combinations & 7 combinations & 2 combinations & 1 combination & 4 combinations \\
\hline
\end{tabular}

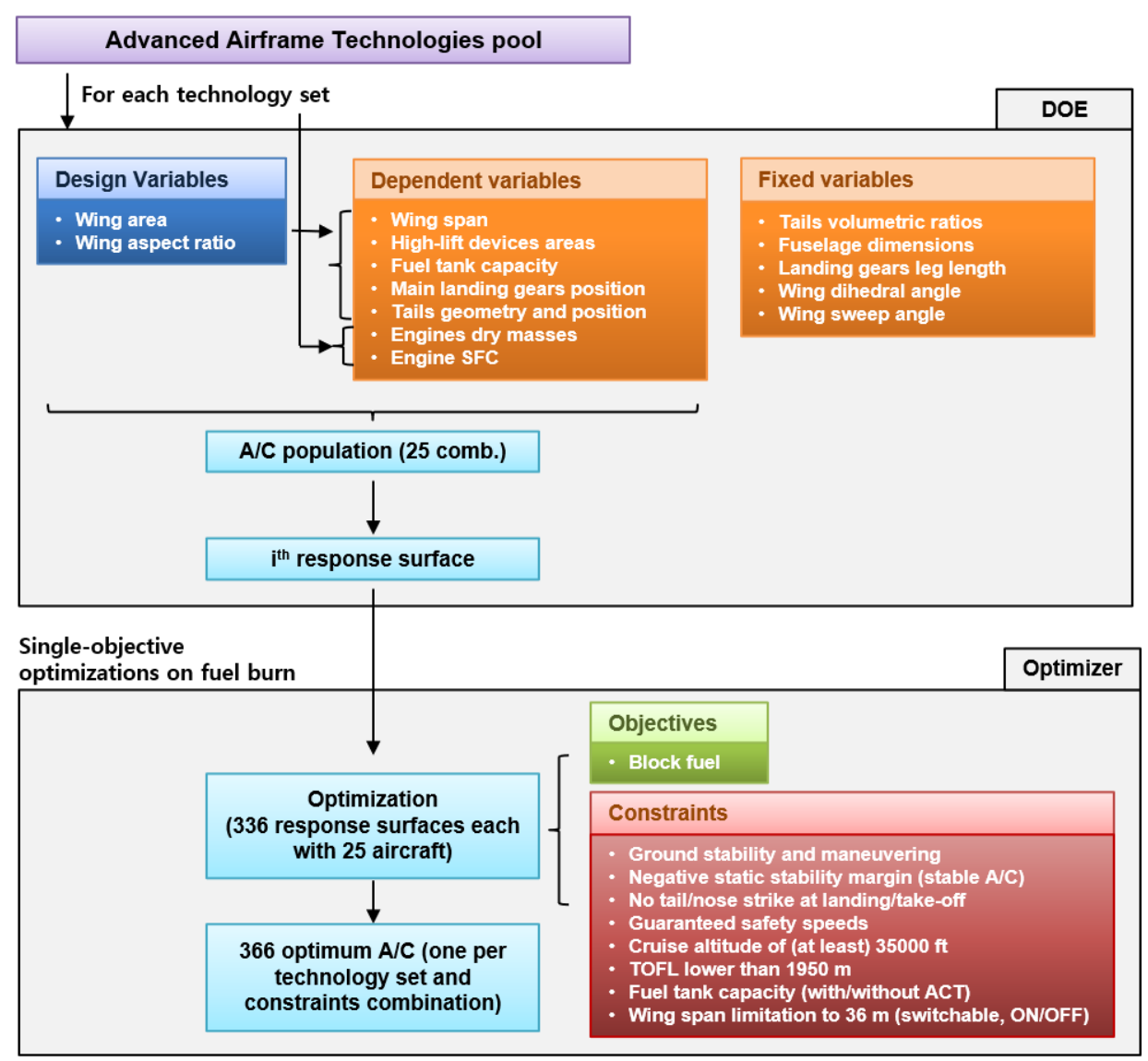

Fig. 6 First step of the optimization for the UM target A/C model. The light blue boxes represent the workflow implemented for a fixed set of constraints.

Following this first step, the results coming from the 1344 optimizations on block fuel have been used to perform a further analysis, aiming at providing, for each combination of constraints, three possible solutions: one for minimum block fuel, one for minimum total DOC, and a balanced solution, representing some sort of compromise between minimum costs and block fuel. For this analysis, each of the optimum aircraft obtained at the previous step has been analyzed again by means of a complete multi-disciplinary analysis cycle (the one reported in Fig. 2), to have more reliable results than those calculated by means of an interpolation of the related response surface (although these 
differences were quite limited). The results of these additional analyses have been reported in Fig. 7-12, and have been summarized in Tables 14-16, with Table 17 providing information on the performance of the baseline configuration. These triplets of "optimum" solutions per constraints combinations have been then compared in terms of block fuel, pollutant emissions $\left(\mathrm{CO}_{2}\right)$, and total DOC with the ADORNO reference UM configuration, to check the effects of the implemented technologies (airframe and engine) on the matching of the ambitious targets set by Clean Sky 2. Independently of the set of constraints adopted for the optimizations, the abovementioned solutions all share the same combinations of advanced airframe technologies. In addition to the more-electric OBS architecture and the advanced powerplant system:

- Advanced materials on both the fuselage and the landing gears for the minimum total DOC solutions;

- HLFC system on the main wing, tails and nacelles, riblets on the fuselage and all the lifting surfaces of the airplane, variable camber wing, and advanced composites and alloys on the fuselage and the landing gears, for the minimum block fuel solutions;

- HLFC system on all the lifting surfaces and the nacelles, along with the use of advanced materials for both the fuselage and the landing gears, for the balanced solutions.

The minimum block fuel solutions, therefore, are also the ones characterized by the highest increase in terms of total DOC with respect to the Y2014 reference model. This is strictly related to the impact that the set of advanced technologies has on the aircraft price and on maintenance costs, according to the implemented modelling described in section IV, despite the provided reduction in terms of block fuel. Nevertheless, in order to comply with the project objectives in terms of emissions reduction, the final choice for the optimum target UM A/C has been made by selecting the minimum block fuel solution for the optimization study with constrained wing span and an additional fuel tank. This solution, as reported in Table 14, guarantees a $-20.12 \%$ reduction in terms of $\mathrm{CO}_{2}$ emissions, which is below the Clean Sky 2 minimum target. Although the results for the optimization study with no limitations on the maximum wing span would have allowed even higher reductions (an additional $-1.42 \%$ for $\mathrm{CO}_{2}$ ) it was concluded that, for an envisaged EIS in the 2030-2035 timeframe, a modification to the current limitations in terms of maximum allowable wing span for category $\mathrm{C}$ airplanes (due to the current size of taxy ways and hangars) would have been excessively unrealistic. Geometric characteristics in terms of wing planform parameters have been reported for the four triplets of best solutions in Fig. 12. Among all the constrained maximum wing span solutions, the selected one is characterized by the lowest wing planform surface combined with the highest allowable aspect ratio.

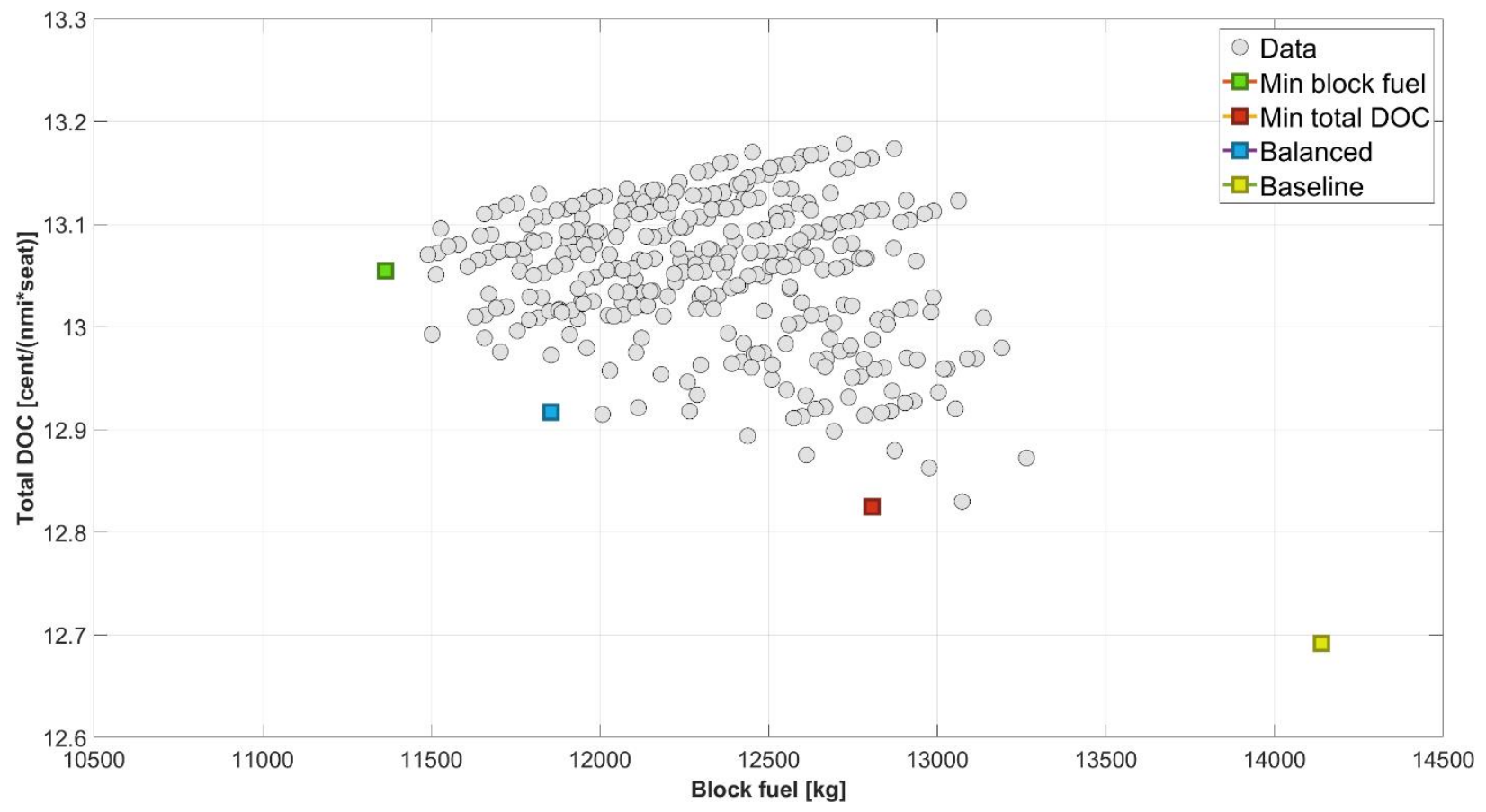

Fig. 7 Results for the optimizations carried out with constraints on both the maximum wing span and ACT availability. Minimum block fuel, minimum total DOC and balanced solutions have been highlighted and compared with the baseline. 


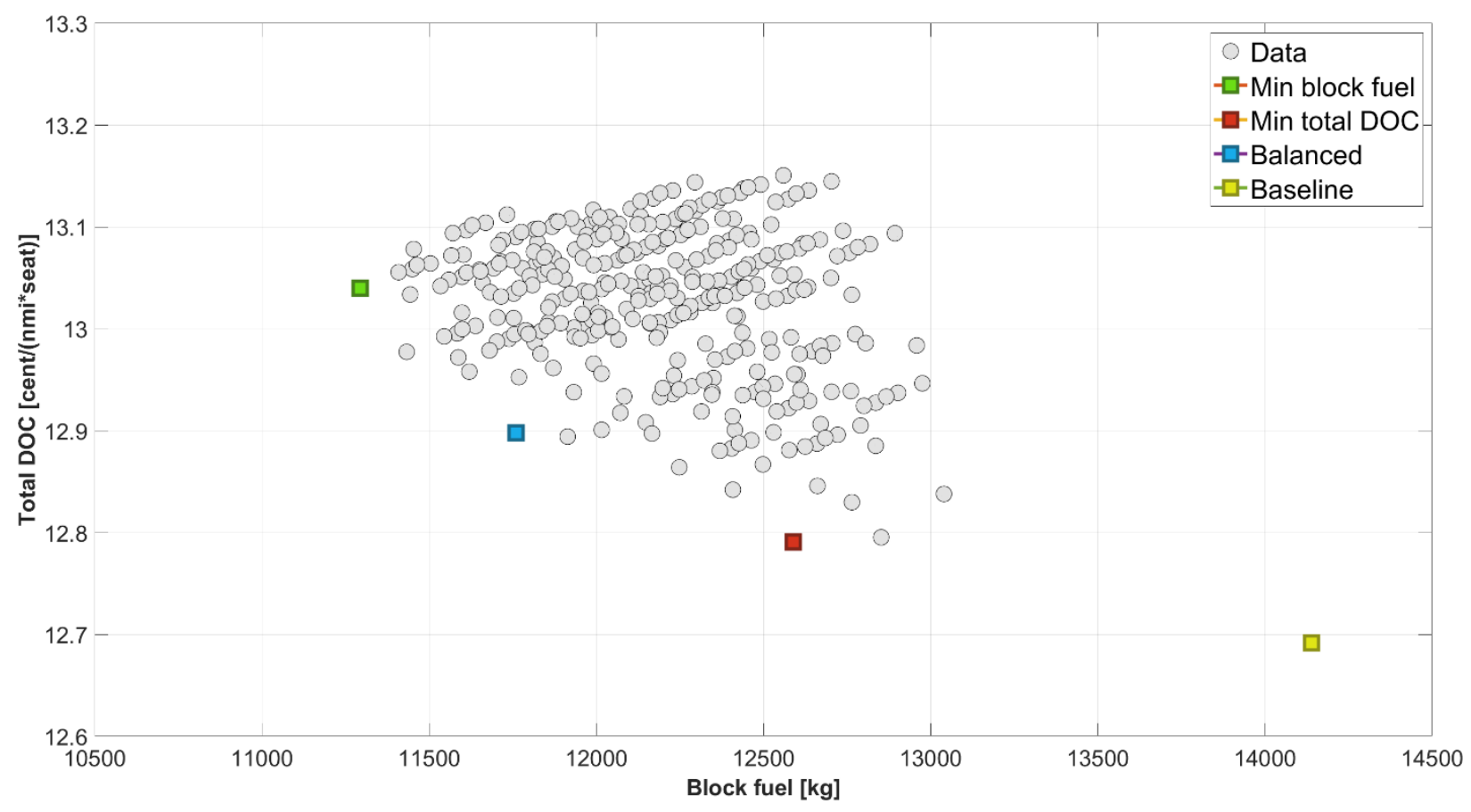

Fig. 8 Results for the optimizations carried out with a constraint on the maximum wing span, with an ACT housed in the fuselage. Minimum block fuel, minimum total DOC and balanced solutions have been highlighted and compared with the baseline.

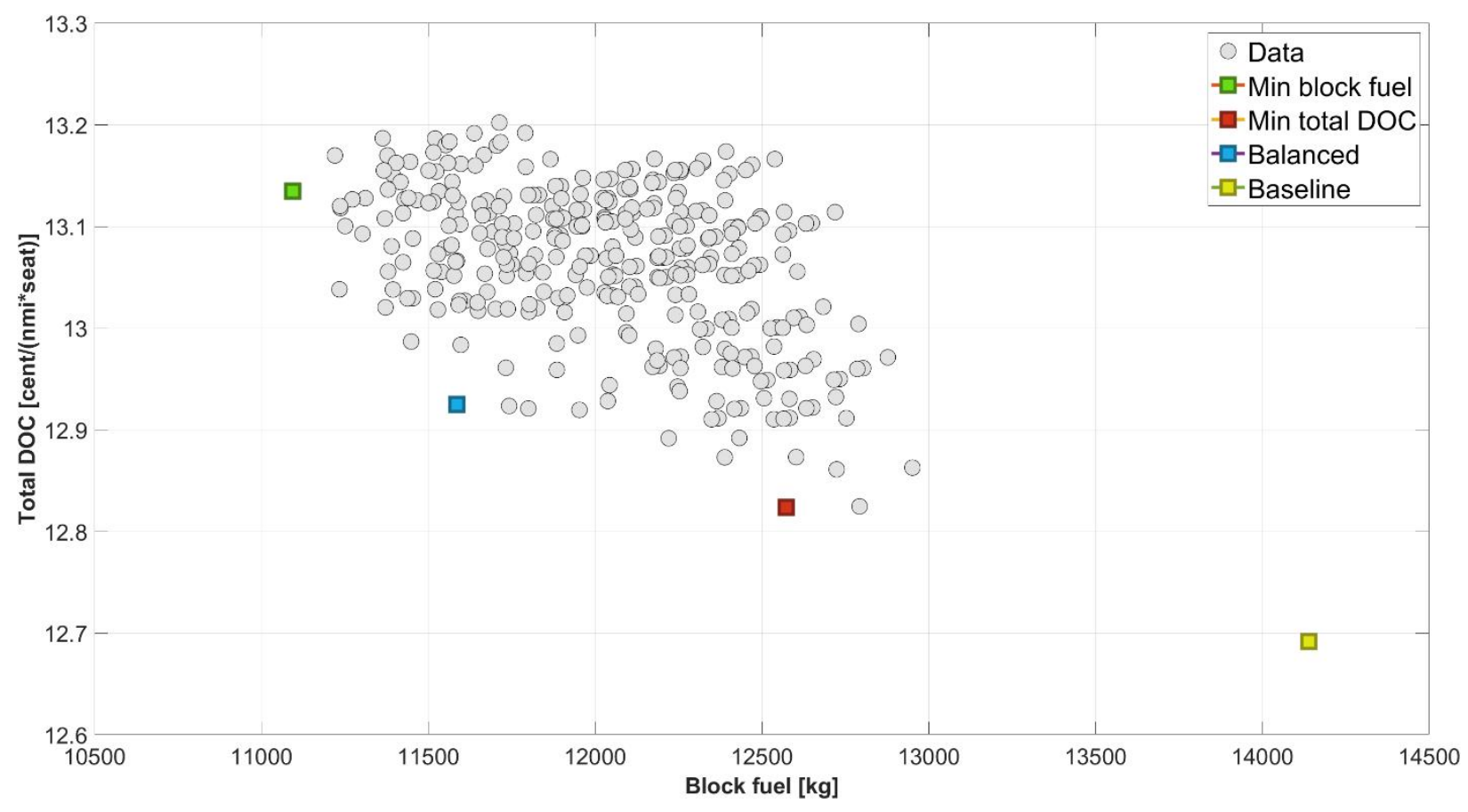

Fig. 9 Results for the optimizations carried out with no constraint on the maximum allowable wing span, but with no ACT housed in the fuselage. Minimum block fuel, minimum total DOC and balanced solutions have been highlighted and compared with the baseline. 


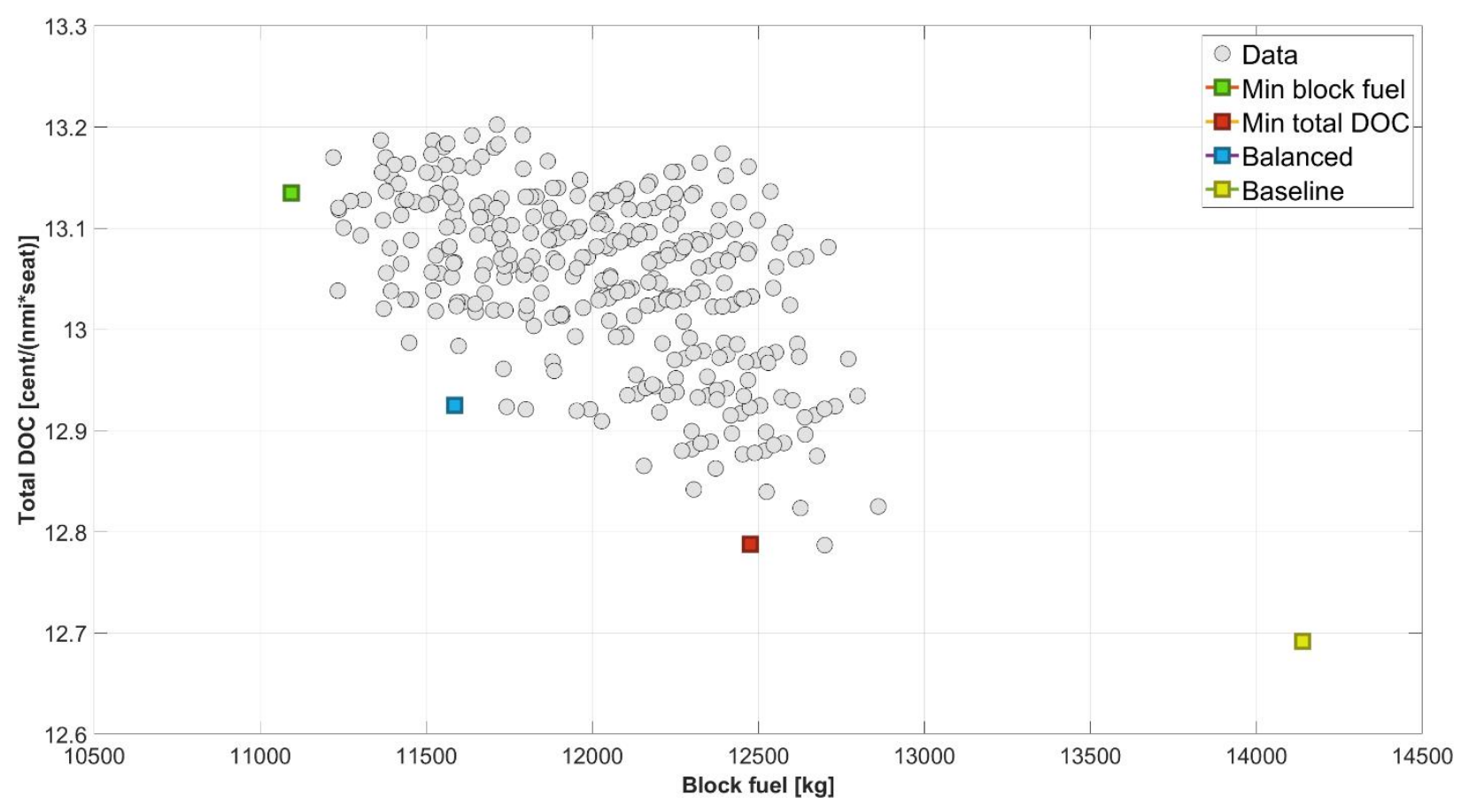

Fig. 10 Results for the optimizations carried out with no constraint on the maximum allowable wing span and housing an ACT in the fuselage. Minimum block fuel, minimum total DOC and balanced solutions have been highlighted and compared with the baseline.

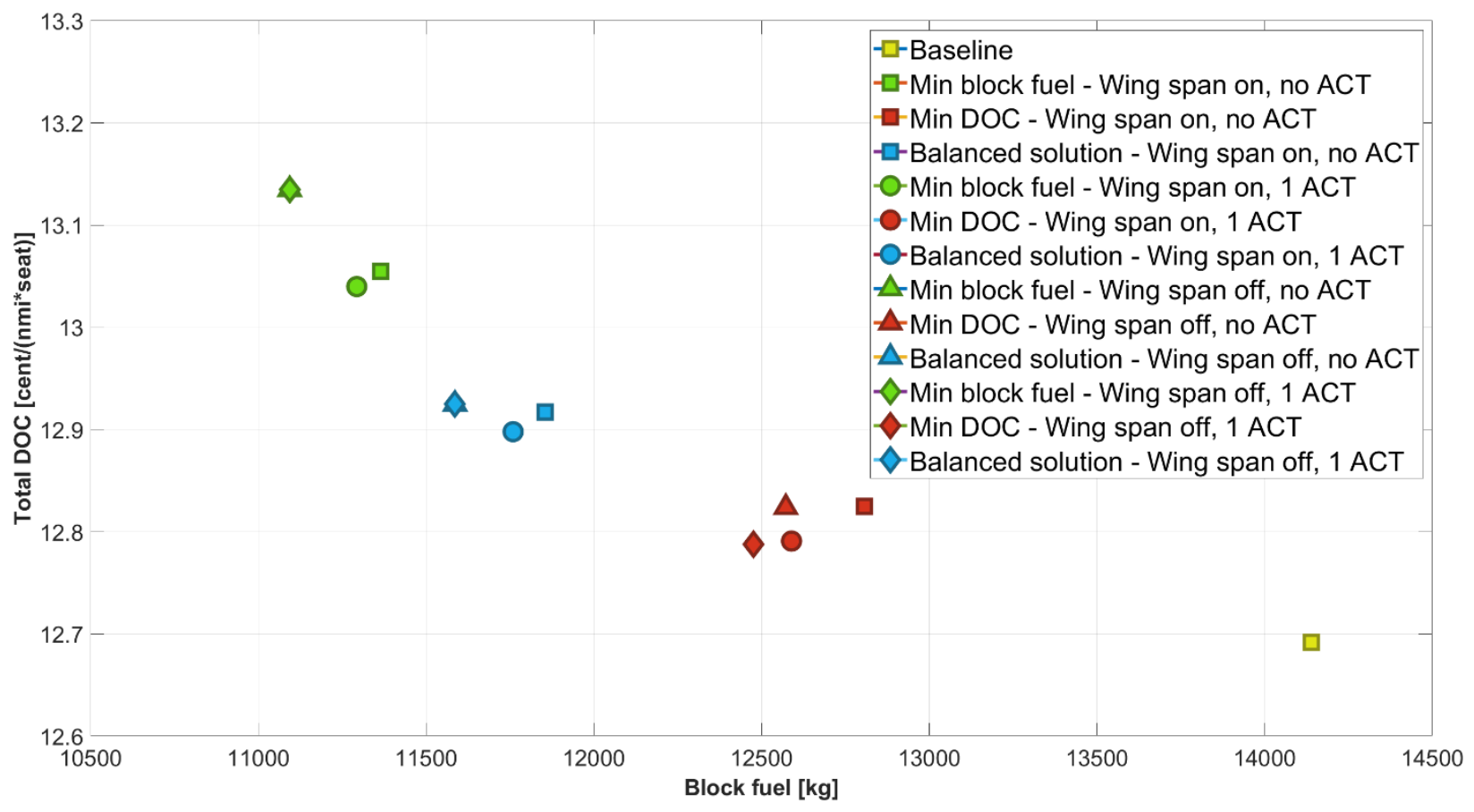

Fig. 11 Comparison between minimum block fuel, minimum total DOC and balanced solutions per optimization constraints permutation with respect to the baseline. 


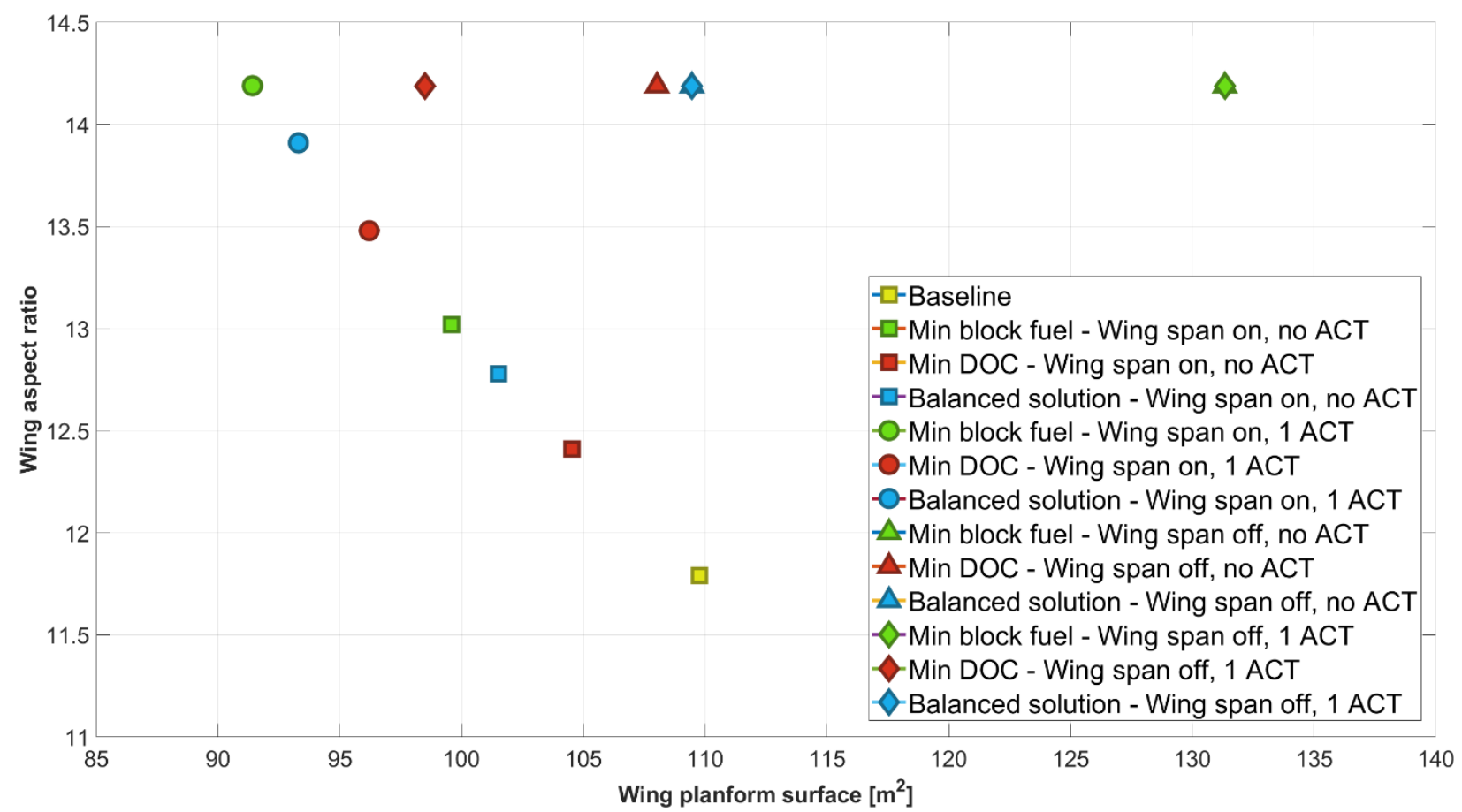

Fig. 12 Geometric characteristics in terms of fuel burn optimization design variables of the minimum block fuel, minimum total DOC and balanced solutions, considering the four different sets of constraints.

Table 14 Minimum block fuel solutions for the four blocks of optimizations carried out.

\begin{tabular}{|c|c|c|c|c|}
\hline \multicolumn{5}{|c|}{ Minimum block fuel solutions - 3100 nmi, 140 pax mission } \\
\hline & \multicolumn{4}{|c|}{ Optimization constraints combinations } \\
\cline { 2 - 5 } & $\begin{array}{c}\text { Active wing span } \\
\text { limitation - 1 ACT }\end{array}$ & $\begin{array}{c}\text { Active wing span } \\
\text { limitation - No ACT }\end{array}$ & $\begin{array}{c}\text { No wing span } \\
\text { limitation - 1 ACT }\end{array}$ & $\begin{array}{c}\text { No wing span } \\
\text { limitation - No ACT }\end{array}$ \\
\hline Block fuel [kg] & 11294 & 11365 & 11094 & 11094 \\
\hline Block fuel $\mathbf{\Delta \%}$ wrt baseline & $\mathbf{- 2 0 . 1 2 \%}$ & $\mathbf{- 1 9 . 6 2 \%}$ & $\mathbf{- 2 1 . 5 4 \%}$ & $\mathbf{- 2 1 . 5 4 \%}$ \\
\hline $\mathbf{C O}_{\mathbf{2}}$ emissions [kg] & 40874 & 41130 & 40147 & 40147 \\
\hline $\mathbf{C O}_{\mathbf{2} \%} \mathbf{\Delta}$ wrt baseline & $\mathbf{- 2 0 . 1 2 \%}$ & $\mathbf{- 1 9 . 6 2 \%}$ & $\mathbf{- 2 1 . 5 4 \%}$ & $\mathbf{- 2 1 . 5 4 \%}$ \\
\hline Total DOC [cent/(nmi*seat)] & 13.040 & 13.055 & 13.135 & 13.135 \\
\hline Total DOC $\mathbf{\Delta \%}$ wrt baseline & $\mathbf{+ 2 . 7 5 \%}$ & $\mathbf{+ 2 . 8 6 \%}$ & $\mathbf{+ 3 . 4 9 \%}$ & $\mathbf{+ 3 . 4 9 \%}$ \\
\hline
\end{tabular}

Table 15 Minimum total DOC solutions for the four blocks optimizations carried out.

\begin{tabular}{|c|c|c|c|c|}
\hline \multicolumn{5}{|c|}{ Minimum total DOC solutions - 3100 nmi, 140 pax mission } \\
\hline & \multicolumn{4}{|c|}{ Optimization constraints combinations } \\
\cline { 2 - 5 } & $\begin{array}{c}\text { Active wing span } \\
\text { limitation - 1 ACT }\end{array}$ & $\begin{array}{c}\text { Active wing span } \\
\text { limitation - No ACT }\end{array}$ & $\begin{array}{c}\text { No wing span } \\
\text { limitation - 1 ACT }\end{array}$ & $\begin{array}{c}\text { No wing span } \\
\text { limitation - No ACT }\end{array}$ \\
\hline Block fuel [kg] & 12590 & 12807 & 12476 & 12573 \\
\hline Block fuel $\mathbf{\Delta \%}$ wrt baseline & $\mathbf{- 1 0 . 9 5 \%}$ & $\mathbf{- 9 . 4 2 \%}$ & $\mathbf{- 1 1 . 7 6 \%}$ & $\mathbf{- 1 1 . 0 7 \%}$ \\
\hline $\mathbf{C O}_{\mathbf{2}}$ emissions [kg] & 45566 & 46349 & 45152 & 45505 \\
\hline $\mathbf{C O}_{\mathbf{2} \boldsymbol{\Delta} \% \text { wrt baseline }}$ & $\mathbf{- 1 0 . 9 5 \%}$ & $\mathbf{- 9 . 4 2 \%}$ & $\mathbf{- 1 1 . 7 6 \%}$ & $\mathbf{- 1 1 . 0 7 \%}$ \\
\hline Total DOC [cent/(nmi*seat)] & $\mathbf{1 2 . 7 9 1}$ & 12.825 & 12.788 & 12.824 \\
\hline Total DOC $\mathbf{\Delta \%}$ wrt baseline & $\mathbf{+ 0 . 7 8 \%}$ & $\mathbf{+ 1 . 0 5 \%}$ & $\mathbf{+ 0 . 7 6 \%}$ & $\mathbf{+ 1 . 0 4 \%}$ \\
\hline
\end{tabular}


Table 16 Balanced solutions (i.e., compromise solutions between minimum block fuel and total DOC) for the four blocks of optimizations carried out.

\begin{tabular}{|c|c|c|c|c|}
\hline \multicolumn{5}{|c|}{ Balanced solutions - 3100 nmi, 140 pax mission } \\
\hline & \multicolumn{4}{|c|}{ Optimization constraints combinations } \\
\cline { 2 - 5 } & $\begin{array}{c}\text { Active wing span } \\
\text { limitation - 1 ACT }\end{array}$ & $\begin{array}{c}\text { Active wing span } \\
\text { limitation - No ACT }\end{array}$ & $\begin{array}{c}\text { No wing span } \\
\text { limitation - 1 ACT }\end{array}$ & $\begin{array}{c}\text { No wing span } \\
\text { limitation - No ACT }\end{array}$ \\
\hline Block fuel [kg] & 11760 & 11855 & 11587 & 11587 \\
\hline Block fuel $\mathbf{\Delta \%}$ wrt baseline & $\mathbf{- 1 6 . 8 2 \%}$ & $\mathbf{- 1 6 . 1 6 \%}$ & $\mathbf{- 1 8 . 0 5 \%}$ & $\mathbf{- 1 8 . 0 5 \%}$ \\
\hline $\mathbf{C O}_{\mathbf{2}}$ emissions [kg] & 42562 & 42900 & 41933 & 41933 \\
\hline $\mathbf{C O}_{\mathbf{2}} \mathbf{\Delta} \%$ wrt baseline & $\mathbf{- 1 6 . 8 2 \%}$ & $\mathbf{- 1 6 . 1 6 \%}$ & $\mathbf{- 1 8 . 0 5 \%}$ & $\mathbf{- 1 8 . 0 5 \%}$ \\
\hline Total DOC [cent/(nmi*seat)] & 12.898 & 12.917 & 12.925 & 12.925 \\
\hline Total DOC $\mathbf{\Delta \%}$ wrt baseline & $\mathbf{+ 1 . 6 3 \%}$ & $\mathbf{+ 1 . 7 7 \%}$ & $\mathbf{+ 1 . 8 4 \%}$ & $\mathbf{+ 1 . 8 4 \%}$ \\
\hline
\end{tabular}

Table 17 Baseline aircraft block fuel and emissions for the mission specified by the set of TLAR presented in Table 1.

\begin{tabular}{|c|c|}
\hline Baseline A/C (reference Y2014) performance - $\mathbf{3 1 0 0 ~} \mathbf{~ n m i}, \mathbf{1 4 0}$ pax mission \\
\hline Block fuel [kg] & 14139 \\
\hline CO2 emissions [kg] & 51169 \\
\hline Total DOC [cent/(nmi*seat)] & 12.692 \\
\hline
\end{tabular}

\section{Conclusions}

This paper has summarized part of the work that has been carried out for the second work package of the Clean Sky 2 project ADORNO, dealing with the design of Y2025+ advanced aircraft models. Activities on the aircraft design side have been carried out by means of an in-house developed framework, implementing multidisciplinary analyses and optimizations by means of an object-oriented approach to the programming of tools, packages and libraries supporting the designer. To comply with the objectives of the project, the abovementioned framework has been enriched with a module specifically dedicated to the inclusion of the effects of evolutionary airframe technologies on those disciplines accounted in the design process (weights, aerodynamics, power off-takes, and costs).

The close collaboration between UNINA and MTU - coordinator and topic manager of the project, respectively and the exchange of data between the partners in terms of fuel burn trade factors for the most relevant engine-related parameters, has allowed the German manufacturer to produce the dataset of performance and emissions for an advanced GTF, to be equipped on the target A/C models to be designed for the project.

An UM Y2014 reference A/C, previously designed for the project, has been subsequently retrofitted with the advanced powerplant system, with only minor modifications to the original geometry of the model, in order to ensure ground stability with minor impact on aircraft performance.

This updated baseline model has been subsequently used as the input in a two-step optimization process, aiming at reducing fuel burn, and thus pollutant emissions. Four optimization blocks have been carried out for different sets of constraints. For each set of constraints, 366 optimum A/C, one for each selected combination of airframe technologies, have been identified, following a single-objective optimization on fuel burn by changing wing planform parameters. These 1344 optimum $\mathrm{A} / \mathrm{C}$ have been subsequently examined, to detect for each combination of constraints the best solutions in terms of block fuel and total DOC, and in-between balanced solutions. Being emissions one of the most relevant topic in the project, in order to comply with the targets sets by the Clean Sky 2 programme, the minimum block fuel solution for the constrained wing span with an additional ACT case has been selected as the final configuration for the ADORNO UM target A/C.

The adopted methodology for the inclusion of the effects of advanced airframe technologies has proved to be robust enough, allowing to obtain results in terms of block fuel comparable to those expected. Results could be further improved by including a more refined modelling of some effects, especially those related to weight penalties and power off-takes. Although not directly required by the project, a low-level estimation of costs deriving from the inclusion of these technologies has been also implemented. Results would surely benefit from a more refined and much more detailed modelling of the problem, which may be addressed by the authors in future works. 


\section{Acknowledgments}

The ADORNO project has received funding from the Clean Sky 2 Joint Undertaking under the European Union's Horizon 2020 research and innovation program under Grant Agreement ENG ITD $n^{\circ}$ 821043. The authors are grateful of MTU support on this research topic. The content of this paper reflects only the author's view and both the European Commission and the Clean Sky 2 Joint Undertaking are not responsible for any use that may be made of the information it contains.
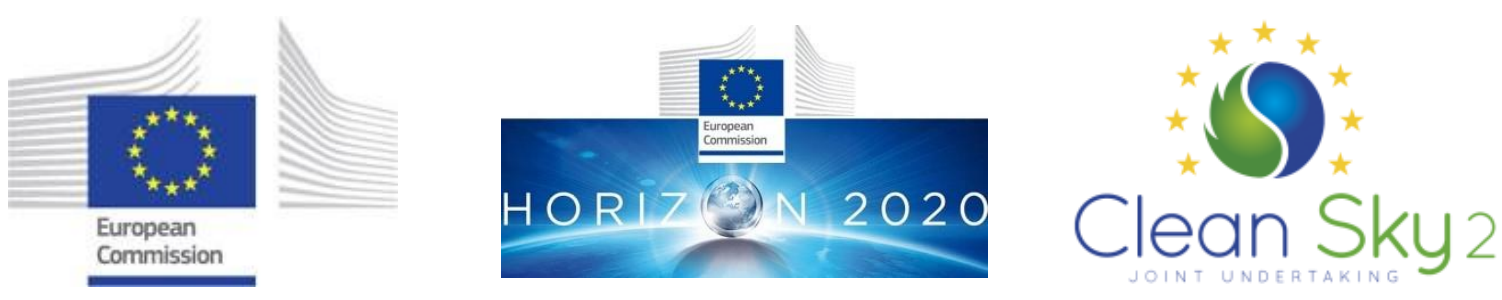

\section{References}

[1] Lefebvre, T., Bartoli, N., Dubreuil, S., Panzeri, M., Lombardi, R., Della Vecchia, P., Stingo, L., Nicolosi, F., De Marco, A., Ciampa, P.D., Anisimov, K., Savelyev, A., Mirzoyan, A., and Isyanov, A., "Enhancing optimization capabilities using the AGILE collaborative MDO framework with application to wing and nacelle design", Progress in Aerospace Sciences, Vol. 119, November 2020, art. 100649, ISSN: 0376-0421. doi: 10.1016/j.paerosci.2020.100649.

[2] Kroo, I., "An interactive system for aircraft design and optimization", In Proceedings of the Aerospace Design Conference, Irvine, CA, USA, 3-6 February 1992, doi: 10.2514/6.1992-1190.

[3] Kroo, I., Altus, S., Braun, R., Gage, P., and Sobieski, I, "Multidisciplinary optimization methods for aircraft preliminary design", In Proceedings of the 5th Symposium on Multidisciplinary Analysis and Optimization, Panama City Beach, FL, USA, 7-9 September 1994, doi: 10.2514/6.1994-4325.

[4] Antoine, N.E., and Kroo, I., "Framework for Aircraft Conceptual Design and Environmental Performance Studies", AIAA Journal, 2005, 43, 2100-2109, doi: 10.2514/1.13017.

[5] Allison, J., Roth, B., Kokkolaras, M., Kroo, I., and Papalambros, P, “Aircraft Family Design Using Decomposition-Based Methods", In Proceedings of the 11th AIAA/ISSMO Multidisciplinary Analysis and Optimization Conference, Portsmouth, VA, USA, 6-8 September 2006, doi: 10.2514/6.2006-6950.

[6] Martins, J.R.R.A., and Marriage, C, “An Object-Oriented Framework for Multidisciplinary Design Optimization", In Proceedings of the 48th AIAA/ASME/ASCE/AHS/ASC Structures, Structural Dynamics, and Materials Conference, Honolulu, HI, USA, 23-26 April 2007, doi: 10.2514/6.2007-1906.

[7] Perez, R.E., Jansen, P.W., and Martins, J.R.R.A., "pyOpt: A Python-based object-oriented framework for nonlinear constrained optimization”, Structural and Multidisciplinary Optimization, 2012, 45, 101-118, doi: 10.1007/s00158-0110666-3.

[8] Liem, R.P., Kenway, G.K.W., and Martins, J.R.R.A, "Multimission Aircraft Fuel-Burn Minimization via Multipoint Aerostructural Optimization", AIAA Journal, 2015, 53, 104-122, doi: 10.2514/1.J052940.

[9] Martins, J.R.R.A., and Lambe, A.B, "Multidisciplinary Design Optimization: A Survey of Architectures", AIAA Journal, 2013, 51, 2049-2075, doi: 10.2514/1.J051895.

[10] Nicolosi, F., Della Vecchia, P., Trifari, V., Di Stasio, M., Marulo, F., De Marco, A., Marciello, V., and Cusati, V., "Noise, Emissions, and Costs trade factors for regional jet platforms using a new software for aircraft preliminary design", AIAA Aviation 2020 Forum, AIAA, Virtual Meeting, 2020, doi: 10.2514/6.2020-2638.

[11] Clean Sky 2, "Joint Technical Programme", March 2015, Brussels, Belgium. url: https://ec.europa.eu/research/participants/data/ref/h2020/other/guide-appl/jti/h2020-guide-techprog-cleansky-ju_en.pdf [retrieved 3 June 2021]

[12] Nicolosi, F., De Marco, A., Attanasio, L., and Della Vecchia, P., "Development of a Java-based framework for aircraft preliminary design and optimization”, AIAA Journal of Aerospace Information Systems, Vol. 13, No. 16, 2016, pp. 234242, doi: 10.2514/1.I010404.

[13] Trifari, V., Ruocco, M., Cusati, V., Nicolosi, F., and De Marco, A., "Java Framework for Parametric Aircraft Design Ground Performance”, Aircraft Engineering and Aerospace Technology (AEAT), Vol. 89, No. 4, 2017, pp. 599-608, doi: 10.1108/AEAT-11-2016-0209.

[14] De Marco, A., Cusati, V., Trifari, V., Ruocco, M., Nicolosi, F. and Della Vecchia, P., "A Java Toolchain of Programs for Aircraft Design", Proceedings of the 6th CEAS Air and Space Conference, Bucharest, Romania, 2017, ISBN: 978973-0-25597-3.

[15] Trifari, V., Ruocco, M., Cusati, V., Nicolosi, F., and De Marco, A., "Multi-disciplinary analysis and optimization Java tool for aircraft design", ICAS 31st Congress of the International Council of the Aeronautical Sciences, Belo Horizonte, Brazil, 2018, ISBN: 9783932182884. 
[16] De Marco, A., Di Stasio, M., Della Vecchia, P., Trifari, V., and Nicolosi, F. "Automatic modeling of aircraft external geometries for preliminary design workflows", Aerospace Science and Technology, Vol. 8, 2020. doi: 10.1016/j.ast.2019.105667.

[17] De Marco, A., Trifari, V., Nicolosi, F. and Ruocco, M., "A Simulation-Based Performance Analysis Tool for Aircraft Design Workflows", Aerospace, 7(11), 155, 2020, doi: 10.3390/aerospace7110155.

[18] Sforza, P., Commercial Airplane Design Principles, Elsevier, 2014, ISBN: 9780124199538.

[19] Torenbeek, E., Synthesis of subsonic airplane design: an introduction to the preliminary design of subsonic general aviation and transport aircraft, with emphasis on layout, aerodynamic design, propulsion and performance, Springer Netherlands, 1982, ISBN: 978-94-017-3202-4, doi: 10.1007/978-94-017-3202-4.

[20] Carichner, G., and Nicolai, L., Fundamentals of Aircraft and Airship Design, AIAA Education Series, AIAA, 2013, ISBN: 978-1-60086-751-4, doi: 10.2514/4.867538.

[21] Raymer, D., Aircraft Design: A Conceptual Approach, 5th ed., AIAA Education Series, AIAA, 2012, ISBN: 9781600869112, doi: 10.2514/4.869112.

[22] Sadraey, M., Aircraft Design: A Systems Engineering Approach, John Wiley \& Sons Inc, 2012, ISBN: 978-1-119953401.

[23] Howe, D., Aircraft conceptual design synthesis, John Wiley \& Sons Inc., 2000, ISBN: 978-1-860-58301-8.

[24] Torenbeek, E., Advanced Aircraft Design: Conceptual Design, Analysis and Optimization of Subsonic Civil Airplanes, John Wiley \& Sons Inc, 2013, ISBN: 978-1-118-56811-8.

[25] Nicolosi, F., Della Vecchia, P., Ciliberti, D., and Cusati, V., "Fuselage Aerodynamic Prediction Methods", Aerospace Science and Technology, Elsevier, Vol. 55, Aug. 2016, pp. 332-343, ISSN: 1270-9638, doi: 10.1016/j.ast.2016.06.012.

[26] Nicolosi, F., Ciliberti, D., Della Vecchia, P., and Corcione, S., "Wind tunnel testing of a generic regional turboprop aircraft modular model and development of improved design guidelines", $36^{\text {th }}$ AIAA Applied Aerodynamics Conference, Atlanta, GA, USA, 25-29 June 2018, doi: 10.2514/6.2018-2855.

[27] Della Vecchia, P., Nicolosi, F., and Ciliberti, D., "Aircraft directional stability prediction method by CFD”, 33rd AIAA Applied Aerodynamic Conference, Dallas, TX, USA, 22-26 June 2015, doi: 10.2514/6.2015-2255.

[28] Nicolosi, F., Ciliberti, D., Della Vecchia, P., and Corcione, S., "Experimental analysis of aircraft directional control effectiveness", Aerospace Science and Technology (AESCTE), Vol. 106, November 2020, art. 106099, Elsevier, doi: 10.1016/j.ast.2020.106099.

[29] Blackwell, J.A. Jr, "A finite step method for calculation of theoretical load distributions for arbitrary lifting-surface arrangements at subsonic speeds", NASA Technical Note, D-5335, 1969.

[30] Ruocco, M., "High-lift and stability issues for innovative transport aircraft configurations in aerodynamic design", Ph.D. Dissertation, Department of Industrial Engineering, University of Naples Federico II, Naples, Italy, April 2020.

[31] Trifari, V., "Development of a Multi-Disciplinary Analysis and Optimization framework and applications for innovative efficient regional aircraft", PhD Dissertation, Department of Industrial Engineering, University of Naples Federico II, Naples, Italy, April 2020

[32] Casale C., Polito T., Trifari V., Di Stasio M., Della Vecchia P., Nicolosi F., and Marulo F., "Implementation of a Noise Prediction Software for Civil Aircraft Applications", XXV International Congress of the Italian Association of Aeronautics and Astronautics, AIDAA, Rome, Italy, 2019.

[33] Engineering Sciences Data Unit (ESDU), “Airframe noise prediction”, Item No. 90023, ESDU International plc, London, UK, Nov. 1990.

[34] Engineering Sciences Data Unit (ESDU), "Evaluation of the attenuation of sound by a uniform atmosphere", Item No. 78002, ESDU International plc, London, UK, Apr. 1998.

[35] Engineering Sciences Data Unit (ESDU), "The correction of measured noise spectra for the effects of ground reflection", Item No. 94035, ESDU International plc, London, UK, Dec. 1995.

[36] Engineering Sciences Data Unit (ESDU), "Estimation of lateral attenuation of air-to-ground jet or turbofan noise in onethird octave bands", ESDU International plc, London, UK, Nov. 1993.

[37] Engineering Sciences Data Unit (ESDU), "Estimation of noise shielding by barriers", Item no. 79011, ESDU International plc, London, UK, November 1993.

[38] Kundu, A.K, Aircraft design, Cambridge University Press, 2010, ISBN: 9780511844652, doi: 10.1017/CBO9780511844652.

[39] Nicolosi, F., Corcione, S., Trifari, V., and De Marco, A., "Design and optimization of a large turboprop aircraft", Aerospace (MDPI), Vol. 8, Issue 5, May 2021, article 132, ISSN 2226-4310, doi: 10.3390/aerospace8050132.

[40] International Air Transport Association (IATA), "Aircraft Technology Roadmap", April 2013.

[41] International Air Transport Association (IATA), “Aircraft Technology Roadmap - Technical Annex”, April 2013.

[42] International Air Transport Association (IATA), "Aircraft Technology Roadmap to 2050", 2018. url: https://www.iata.org/contentassets/8d19e716636a47c184e7221c77563c93/technology20roadmap20to20205020no20for eword.pdf [retrieved 3 June 2021]

[43] Joslin, R.D., "Overview of Laminar Flow Control”, NASA/TP-1998-208705, October 1998, Langley Research Center, Hampton, Virginia.

[44] Schmitt, V., Archambaud, J.P., Horstmann, K.H., and Quast, A., "Hybrid Laminar Fin Investigations", RTO AVT Symposium, 8-11 May 2000, Braunschweig, Germany. 
[45] Meyer, P., “Application of HLF Technology to Civil Nacelle”, Proceedings of the CEAS/DragNet European Drag Reduction Conference, edited by P. Thiede, vol. 76, Springer-Verlag, Berlin, 2001, pp. 107-114.

[46] Lange, R.H., "Application of Hybrid Laminar Flow Control to Global Range Military Transport Aircraft", NASA Contractor Report 181638, 1988.

[47] Young, T.M., and Fielding J.P., "Flight Operational Assessment of Hybrid Laminar Flow Control (HLFC) Aircraft", Proceedings of the CEAS/DragNet European Drag Reduction Conference, edited by P. Thiede, vol. 76, Springer-Verlag, Berlin, 2001, pp. 99-106.

[48] Catalano, P., de Rosa, D., Mele, B., Tognaccini, R., Moens, F., "Effects of riblets on the performances of a regional aircraft configuration in NLF conditions", AIAA SciTech Forum, Kissimmee, Florida, 8-12 January 2018.

[49] McLean, J.D., George-Falvy, D.N., and Sullivan, P.P., "Flight-test of turbulent skin friction reduction by riblets", Proceedings of International Conference on Turbulent Drag Reduction by Passive Means, Section 16, London Royal Aeronautical Society, 1987.

[50] Walsh, M., Sellers, W., and McGinley, C., "Riblet drag reduction at flight conditions", $6^{\text {th }}$ Applied Aerodynamics Conference, Williamsburg, Virginia, June 1988.

[51] Coustols, E., Schmitt, V., "Synthesis of experimental riblets studies in transonic conditions", Proceedings of the 45" European Drag Reduction Meeting, Kluwer Academic Publishers, Netherlands, 1990, pp. 123-140.

[52] Carter, D.L., Osborn, R.F., Hetrick, J.A., and Kota, S., "The Quest for Efficient Transonic Cruise", AIAA Aviation Technology Integration and Operations Conference, Belfast, Northern Ireland, 18-20 September 2007.

[53] Lyu, Z., and Martins, J.R.R.A., “Aerodynamic Shape Optimization of an Adaptive Morphing Trailing-Edge Wing”, Journal of Aircraft, Vol. 52, No. 6, 2015.

[54] Fioriti, M., Boggero, L., Mirzoyan, A., and Isyanov, A., "Studies on propulsion and on-board systems matching in AGILE project distributed collaborative MDO environment applying for advanced regional and medium haul jet", $31^{\text {st }}$ Congress of the International Council of the Aeronautical Sciences (ICAS), Belo Horizonte, Brazil, 9-14 September 2018.

[55] Achtembosch, M., "Material flow analysis - A comparison of manufacturing, use and fate of CFRP-Fuselage components versus Aluminum-Components for commercial airliners", Fresenius Environmental Bulletin, January 2003.

[56] Soutis, C., "Carbon fiber reinforced plastics in aircraft construction", Materials Science and Engineering: A, Vol. 412, Issues 1-2, 5 December 2005, pp. 171-176.

[57] Gialanella, S., and Malandruccolo, A., “Aerospace Alloys”, Springer Nature, 2019, ISBN: 978-3-030-24440-8.

[58] Currey, N.S., "Aircraft landing gear design: principles and practices", AIAA Education Series, AIAA, 1988, ISBN: 978-0930403416.

[59] Horonjeff, R., and McKelvey, F.X, "Planning and Design of Airports", McGraw-Hill Education, 1994, ISBN: 9780071446419. 